\title{
Generalized Equalization Algorithm Utilizing Improper ISI
}

\author{
Pei Xiao \\ The Institute of Electronics, Communications and Information Technology \\ Queen's University Belfast, BT3 9DT, United Kingdom \\ E-mail: pei.xiaodecit.qub.ac.uk \\ Rolando Carrasco \\ School of Electrical, Electronic and Computer Engineering \\ University of Newcastle Upon Tyne NE1 7RU, United Kingdom \\ E-mail: r.carrasco@ncl.ac.uk \\ Ian Wassell \\ Digital Technology Group, Computer Laboratory, University of Cambridge \\ 15 JJ Thomson Avenue, CB3 0FD, United Kingdom \\ E-mail: ijw24@eng.cam.ac.uk
}

\begin{abstract}
The improper nature of the intersymbol interference (ISI) for signals transmitted over frequency selective channels is investigated in this paper, and our analysis reveals that for real signals, the improperness originates from both improper signal modulation and the interference cancellation process; whereas for most complex signals, the improperness is only a characteristic of the residual ISI due to interference cancellation. In order to utilize the improperness of ISI, a multistage widely linear equalization algorithm is introduced, and it is generally applicable for both real and complex signal constellations. The results reveal that accounting for the improper nature of the ISI at both the input and output of the equalizer leads to a noticeable performance gain compared to conventional equalization schemes.
\end{abstract}

\section{INTRODUCTION}

For a complex random vector $\mathbf{r}$, its second-order statistics are completely characterized by its autocorrelation matrix $\mathbf{R}=\mathrm{E}\left[\mathbf{r r}^{H}\right]$ as well as the pseudo-autocorrelation matrix $\tilde{\mathbf{R}}=\mathrm{E}\left[\mathbf{r r}^{T}\right]$ [1]. Note that throughout this paper, the superscript operators ()$^{H},()^{*},()^{T}$ denote the conjugate transpose, conjugate, and transpose operations, respectively; and $\mathrm{E}[\cdot]$ denotes expectation operation. Most existing studies on receiver algorithms only exploit the information contained in the autocorrelation function of the observed signal. The pseudo-autocorrelation matrix $\tilde{\mathbf{R}}$ is usually not considered and is implicitly assumed to be zero. While this is the optimum strategy when dealing with proper complex random processes (i.e., when the pseudo-autocorrelation $\tilde{\mathbf{R}}$ is vanishing) [2], it turns out to be sub-optimum in situations where the transmitted signals and/or interference are improper complex random processes (i.e., $\tilde{\mathbf{R}}$ is non-vanishing), for which the performance of a linear receiver can generally be improved by the use of widely linear processing (WLP) [3] ${ }^{1}$. It was shown in [1],

\footnotetext{
${ }^{1}$ There are some exceptions, e.g., as pointed out in [4], coherent detection of BPSK signals with known phase shifts and signature waveforms, for which the use of widely linear filters is unnecessary, since the real part of the signal is a sufficient statistic after phase correction.
}

[5], [6] that significant performance gain can be achieved by applying WLP compared to conventional processing.

The application of WLP in communication systems was first developed for improving the performance of direct sequence code division multiple access (DS-CDMA) systems with improper data modulation, such as pulse-amplitude modulation (PAM), offset quadrature phase shift keying (OQPSK), offset quadrature amplitude modulation (OQAM), and binary phaseshift-keying (BPSK), etc.. For example, it was shown in [7] that for systems employing BPSK modulation, full exploitation of the available information on the second-order statistics of the observations entails the use of WLP and yields a scheme that outperforms the other schemes currently known in the literature. It was shown in [8] that OQPSK modulation generates improper complex multiple access interference (MAI) and the application of a widely linear (WL) receiver to the OQPSK modulated CDMA system yields remarkable output signal-tonoise ratio (SNR) gains over the strictly linear receiver. A WL minimum-output-energy (MOE) receiver is derived based on a modified cost function in [9]. By exploiting the additional information contained in pseudo-covariance matrix of observations, a performance gain can be attained for improper DSCDMA signals. In [10], a new WL zero forcing (ZF) receiver was proposed for multi-carrier transmission systems to combat narrow-band interference (NBI). It was designed under the MOE criterion, and resulted in a substantial improvement over the conventional linear $\mathrm{ZF}$ and minimum mean square error (MMSE) receivers, and in the meantime, led to improved blind channel identification capabilities by exploiting the noncircularity property of the desired signal. In [11], [12], WLP was applied to direct sequence spreading ultra-wideband (DSUWB) systems with BPSK and 4-ary bi-orthogonal keying (4BOK) modulation schemes, respectively. Simulation and analytical results showed that the proposed multiple-input, multiple-output (MIMO) WL equalizers allow for powerefficient DS-UWB transmission close to the matched filter 
bound with moderate computational complexity. Widely linear reception strategies were extended to layered space-time wireless communications in [13], where improved versions of the linear decorrelating, MMSE and nonlinear nulling receivers were developed and analyzed. It was concluded that the proposed receivers not only achieve better performance compared to the conventional receivers, but are also less sensitive to the channel estimation errors. The results also indicate that widely linear detection permits operation even when the number of transmit antennas exceeds the number of receive antennas, and also that widely linear reception of an $M$-ary real constellation outperforms linear reception of an $M$-ary complex constellation.

The application of WLP to complex modulation schemes has been addressed in several papers, e.g., in [14], [15] where WLP was applied to complex signals which become improper due to the use of space-time block coding or widely linear space-time mapping. A novel iterative multiuser detector for DS-CDMA systems with complex modulation schemes was proposed in [16]. Owing to the fact that the residual MAI becomes improper when soft decision feedback is used to cancel the MAI, the use of WLP achieves significant gains in power efficiency and improves convergence speed.

The concept of WLP has been applied in several papers for enhancing the performance of equalizers for combating the ISI induced by frequency-selective multipath channels. For example, the equalization of real-valued data transmitted over ISI channels having complex-valued channel coefficients was considered in [5]; and the equalization of space-time block encoded transmissions over MIMO channels was presented in [14] and [15]. An MMSE equalizer and a decision-feedback equalizer (DFE) employing WLP and implemented via finite impulse response (FIR) filters for a MIMO frequency selective channel have been proposed in [17] and [18]. It was concluded that the use of WLP yields considerable performance improvements at the cost of only a limited increase in complexity compared to conventional linear processing.

Linear MMSE filter based turbo equalization which combines equalization and decoding in an iterative fashion has previously been studied, e.g., in [19]-[21] where only the information contained in the autocorrelation function of the observations was employed. In this paper, we further develop the algorithm presented in [19]-[21] by utilizing the improperness of the ISI and exploiting the information contained in the pseudo-autocorrelation function of the observations. The algorithm introduced in [16] approximates the WL filter output as a proper random process. Our investigation reveals that this might be an appropriate assumption for multiuser detection in presence of MAI, it, however, leads to a sub-optimum solution when dealing with ISI introduced by frequencyselective channels, and our results show that exploitation of the improperness of the interference-plus-noise at the filter output further improves the system performance. The proposed equalization algorithms can be applied to systems with real or complex modulation schemes, and they are shown to outperform the WL MMSE and WL DFE introduced in [5] for real signals; and outperform the linear MMSE scheme introduced in [19]-[21] for complex signals.
The remainder of this paper is organized as follows. Section II describes how improper ISI is utilized in the equalization design; while in Section III, the proposed schemes are evaluated and compared to the conventional schemes over some static and time-varying ISI channels. Finally, in Section IV, conclusions are drawn based on the simulation results.

\section{EQUALIZATION USING IMPROPER ISI}

The transmission system under study will now be briefly described. For a system without channel coding, the information bit sequence is directly mapped into PSK/QAM symbols $\left\{s_{n}\right\}$, which are transmitted over a multipath frequency selective fading channel with $L$ resolvable paths, having complex channel gains $h_{0}, h_{1}, \ldots, h_{L-1}$. The received signal can be expressed as

$$
r_{n}=\sum_{l=0}^{L-1} s_{n-l} h_{l}+v_{n},
$$

where $h_{l}$ is the complex channel coefficient and is assumed to remain constant during the transmission of one block of data. The complex additive white Gaussian noise (AWGN) $v_{n}$ with zero mean and variance $N_{0}$, is assumed to be proper. The proposed equalization algorithms work for both real and complex signals. For simplicity, we have selected BPSK/QPSK as the example real/complex-valued modulation schemes to use in this work. However, the extension of the proposed schemes to higher level amplitude-shift keying (ASK) and PSK/QAM schemes is straightforward. The transmitted symbol at time instant $n$ is denoted as $s_{n}$. For BPSK modulation, $s_{n}= \pm 1$ is real-valued; for QPSK modulation, we denote $s_{n}=x_{n}+j y_{n}$, where $x_{n}, y_{n}=\frac{ \pm 1}{\sqrt{2}}$.

The task of the receiver is to detect the transmitted information bits given the received observation $\left\{r_{n}\right\}$. To this end, we need first to detect the transmitted symbols $\left\{s_{n}\right\}$ which are corrupted with ISI and AWGN. An equalizer is required to reduce the detrimental effect of ISI. Here, we consider the equalization algorithm presented in [19]-[21] and develop an enhanced scheme by modifying the MMSE filter design criterion and applying WLP. Let us define the channel matrix

$$
\mathbf{H}=\left[\begin{array}{cccccccc}
h_{L-1} & h_{L-2} & \ldots & h_{0} & 0 & 0 & \ldots & 0 \\
0 & h_{L-1} & \ldots & h_{1} & h_{0} & 0 & \ldots & 0 \\
\vdots & \vdots & \vdots & & & & & \\
0 & 0 & 0 & \ldots & h_{L-1} & h_{L-2} & \ldots & h_{0}
\end{array}\right]
$$

and the vectors

$$
\begin{aligned}
& \mathbf{s}_{n}=\left[\begin{array}{lllllll}
s_{n-L+1} & \ldots & s_{n-1} & s_{n} & s_{n+1} & \ldots & s_{n+L-1}
\end{array}\right]^{T} ; \\
& \overline{\mathbf{s}}_{n}=\left[\begin{array}{lllllll}
\bar{s}_{n-L+1} & \ldots & \bar{s}_{n-1} & 0 & \bar{s}_{n+1} & \ldots & \bar{s}_{n+L-1}
\end{array}\right]^{T} ; \\
& \mathbf{r}_{n}=\left[\begin{array}{llll}
r_{n} & r_{n+1} & \ldots & r_{n+L-1}
\end{array}\right]^{T} ; \\
& \mathbf{v}_{n}=\left[\begin{array}{llll}
v_{n} & v_{n+1} & \ldots & v_{n+L-1}
\end{array}\right]^{T},
\end{aligned}
$$

where $\mathbf{r}_{n}, \mathbf{v}_{n}$ denote the received vector and the noise vector, respectively; and $\overline{\mathbf{s}}_{n}$ contains the estimate of the interference symbols from the previous iteration. The derivation of $\overline{\mathbf{s}}_{n}$ will 
be given later on. According to (1), the received vector after interference cancellation is given as [19], [20]

$$
\mathbf{r}_{n}^{\prime}=\mathbf{r}_{n}-\mathbf{H} \overline{\mathbf{s}}_{n}=\mathbf{H}\left[\mathbf{s}_{n}-\overline{\mathbf{s}}_{n}\right]+\mathbf{v}_{n}
$$

where $\mathbf{r}_{n}^{\prime}$ is the ISI canceled version of $\mathbf{r}_{n}$. Note that (3) represents a decision-directed iterative scheme, where the detection procedure at the the $n^{\text {th }}$ stage uses the symbol estimates from the $(n-1)^{t h}$ stage. The performance is improved in an iterative manner owing to the fact that the symbols are more accurately estimated (meaning better interference cancellation), as the iteration procedure goes on. For simplicity, the iteration index is omitted, whenever no ambiguity arises.

In order to further suppress the residual interference in $\mathbf{r}_{n}^{\prime}$, an instantaneous linear MMSE filter is applied to $\mathbf{r}_{n}^{\prime}$, to obtain $z_{n}=\mathbf{w}_{n}^{H} \mathbf{r}_{n}^{\prime}$, where the filter coefficient vector $\mathbf{w}_{n}$ is chosen to minimize $e_{n}^{\mathrm{L}}=\mathrm{E}\left\{\left|\mathbf{w}_{n}^{H} \mathbf{r}_{n}^{\prime}-s_{n}\right|^{2}\right\}$. Refer to [19]-[21] for a detailed description of the conventional MMSE algorithm. Next, we shall discuss how the performance can be improved by applying WLP, the principle of which is not only to process $\mathbf{r}_{n}^{\prime}$, but also its conjugated version $\mathbf{r}_{n}^{* *}$ in order to derive the filter output, i.e.,

$$
z_{n}=\boldsymbol{\omega}_{n}[0] \mathbf{r}_{n}^{\prime}+\boldsymbol{\omega}_{n}[1] \mathbf{r}_{n}^{* *}=\boldsymbol{\omega}_{n}^{H} \mathbf{y}_{n}
$$

where $\boldsymbol{\omega}_{n}=\left[\begin{array}{ll}\boldsymbol{\omega}_{n}[0] & \boldsymbol{\omega}_{n}[1]\end{array}\right]^{H}$ and $\mathbf{y}_{n}=\left[\begin{array}{ll}\mathbf{r}_{n}^{\prime T} & \mathbf{r}_{n}^{\prime H}\end{array}\right]^{T}$. Substituting (3) into the above equation yields

$$
\begin{aligned}
z_{n}= & \boldsymbol{\omega}_{n}[0] \mathbf{H}\left[\mathbf{s}_{n}-\overline{\mathbf{s}}_{n}\right]+\boldsymbol{\omega}_{n}[0] \mathbf{v}_{n}+\boldsymbol{\omega}_{n}[1] \mathbf{H}^{*}\left[\mathbf{s}_{n}^{*}-\overline{\mathbf{s}}_{n}^{*}\right] \\
& +\boldsymbol{\omega}_{n}[1] \mathbf{v}_{n}^{*}=\boldsymbol{\omega}_{n}[0] \mathbf{h} s_{n}+\boldsymbol{\omega}_{n}[1] \mathbf{h}^{*} s_{n}^{*}+\mathbf{v}_{n}^{\prime}
\end{aligned}
$$

where $\mathbf{v}_{n}^{\prime}=\boldsymbol{\omega}_{n}[0] \mathbf{v}_{n}+\boldsymbol{\omega}_{n}[1] \mathbf{v}_{n}^{*}$, and $\mathbf{h}=$ $\left[\begin{array}{llll}h_{0} & h_{1} & \ldots & h_{L-1}\end{array}\right]^{T}$. The decision statistic $z_{n}$ contains the scaled version of the symbol $s_{n}$ and its conjugate $s_{n}^{*}$ as well as the combined interference cancellation residual and noise denoted as $\mathbf{v}_{n}^{\prime}$. Accounting for this changes at the filter output $z_{n}$, the cost function needs to be revised accordingly as

$$
e_{n}^{\mathrm{WL}}=\mathrm{E}\left[\left|z_{n}-s_{n}-\gamma s_{n}^{*}\right|^{2}\right]=\mathrm{E}\left[\left|\boldsymbol{\omega}_{n}^{H} \mathbf{y}_{n}-s_{n}-\gamma s_{n}^{*}\right|^{2}\right] .
$$

For real-valued constellations (the symbol $s_{n}$ is real-valued), $\gamma=1$ is appropriate so that $e_{n}^{\mathrm{WL}}=\mathrm{E}\left\{\left|\boldsymbol{\omega}_{n}^{H} \mathbf{y}_{n}-s_{n}-s_{n}^{*}\right|^{2}\right\}=$ $\mathrm{E}\left\{\left|\boldsymbol{\omega}_{n}^{H} \mathbf{y}_{n}-2 s_{n}\right|^{2}\right\}$. In this case, $\boldsymbol{\omega}_{n}[2]=\boldsymbol{\omega}_{n}^{*}[1]$, and therefore, $e_{n}^{\mathrm{WL}}=\mathrm{E}\left\{\left|2 \operatorname{Re}\left\{\boldsymbol{\omega}_{n}[1] \mathbf{r}_{n}^{\prime}\right\}-2 s_{n}\right|^{2}\right\}=\mathrm{E}\left\{4 \mid \operatorname{Re}\left\{\boldsymbol{\omega}_{n}[1] \mathbf{r}_{n}^{\prime}\right\}-\right.$ $\left.\left.s_{n}\right|^{2}\right\}$ (where $\operatorname{Re}\{\cdot\}$ and $\operatorname{Im}\{\cdot\}$ denote the real and imaginary part of a complex variable, respectively), which is a better cost function than $e_{n}^{\mathrm{L}}=\mathrm{E}\left\{\left|\mathbf{w}_{n}^{H} \mathbf{r}_{n}^{\prime}-s_{n}\right|^{2}\right\}$, since a conventional MMSE filter yields a complex-valued filter output; however, only the real part of this output is relevant for the decision for systems with a real-valued constellation. It was shown in [5] that $e_{n}^{\mathrm{WL}}<e_{n}^{\mathrm{L}}$, leading to an equalizer with enhanced performance. For complex-valued constellations, the role of the parameter $\gamma$ (the value of which should not be 1) will become apparent in Section III. It is worth noticing that the conventional linear MMSE equalizer is a special case of the WL equalizer, when $\boldsymbol{\omega}_{n}[1]=\mathbf{w}_{n}^{H}$ and $\boldsymbol{\omega}_{n}[2]=\mathbf{0}$. The WL equalizers are expected to exhibit better performance than their linear counterparts. In particular, the conditions under which
WL estimator can yield significant improvements over linear ones are detailed in [3]. By expanding (4), we obtain

$$
\begin{aligned}
e_{n}^{\mathrm{WL}}= & \mathrm{E}\left[\left|\boldsymbol{\omega}_{n}^{H} \mathbf{y}_{n}-s_{n}-\gamma s_{n}^{*}\right|^{2}\right] \\
= & \mathrm{E}\left[\left(\boldsymbol{\omega}_{n}^{H} \mathbf{y}_{n}-s_{n}-\gamma s_{n}^{*}\right)\left(\mathbf{y}_{n}^{H} \boldsymbol{\omega}_{n}-s_{n}^{*}-\gamma s_{n}\right)\right] \\
= & \left\{\begin{array}{cc}
\boldsymbol{\omega}_{n}^{H} \mathbf{C}_{n} \boldsymbol{\omega}_{n}-\gamma \boldsymbol{\omega}_{n}^{H} \tilde{\mathbf{C}}_{\mathbf{y} s}-\boldsymbol{\omega}_{n}^{H} \mathbf{C}_{\mathbf{y} s}-\mathbf{C}_{s \mathbf{y}} \boldsymbol{\omega}_{n} \\
-\gamma \tilde{\mathbf{C}}_{s \mathbf{y}}^{*} \boldsymbol{\omega}_{n}+\left(1+2 \gamma+\gamma^{2}\right) \sigma_{s}^{2} ; & \text { for BPSK } \\
\boldsymbol{\omega}_{n}^{H} \mathbf{C}_{n} \boldsymbol{\omega}_{n}-\gamma \boldsymbol{\omega}_{n}^{H} \tilde{\mathbf{C}}_{\mathbf{y} s}-\boldsymbol{\omega}_{n}^{H} \mathbf{C}_{\mathbf{y} s}-\mathbf{C}_{s \mathbf{y}} \boldsymbol{\omega}_{n} \\
-\gamma \tilde{\mathbf{C}}_{s \mathbf{y}}^{*} \boldsymbol{\omega}_{n}+\left(1+\gamma^{2}\right) \sigma_{s}^{2} ; & \text { for QPSK }
\end{array}\right.
\end{aligned}
$$

where $\sigma_{s}^{2}$ denotes the average symbol energy and

$$
\begin{aligned}
& \mathbf{C}_{n}=\mathrm{E}\left\{\mathbf{y}_{n} \mathbf{y}_{n}^{H}\right\}=\mathrm{E}\left\{\left[\begin{array}{c}
\mathbf{r}_{n}^{\prime} \\
\mathbf{r}_{n}^{\prime *}
\end{array}\right]\left[\begin{array}{ll}
\mathbf{r}_{n}^{\prime H} & \mathbf{r}_{n}^{\prime T}
\end{array}\right]\right\}=\left[\begin{array}{cc}
\mathbf{R}_{n} & \tilde{\mathbf{R}}_{n} \\
\tilde{\mathbf{R}}_{n}^{*} & \mathbf{R}_{n}^{*}
\end{array}\right] \\
& =\left[\begin{array}{cc}
\mathbf{H} \mathbf{V}_{n} \mathbf{H}^{H}+N_{0} \mathbf{I} & \mathbf{H} \tilde{\mathbf{V}}_{n} \mathbf{H}^{T} \\
\mathbf{H}^{*} \tilde{\mathbf{V}}_{n}^{*} \mathbf{H}^{H} & \mathbf{H}^{*} \mathbf{V}_{n} \mathbf{H}^{T}+N_{0} \mathbf{I}
\end{array}\right] ; \\
& \mathbf{V}_{n}=\operatorname{diag}\left\{\left[1-\left|\bar{s}_{n-L+1}\right|^{2} \quad \ldots \quad 1-\left|\bar{s}_{n-1}\right|^{2} \quad 1\right.\right. \\
& \left.\left.1-\left|\bar{s}_{n+1}\right|^{2} \quad \ldots \quad 1-\left|\bar{s}_{n+L-1}\right|^{2}\right]\right\} \\
& \mathbf{C}_{\mathbf{y} s}=\mathbf{C}_{s \mathbf{y}}^{H}=\mathrm{E}\left\{\mathbf{y}_{n} s^{*}\right\}=\mathrm{E}\left\{\left[\begin{array}{c}
\mathbf{r}_{n}^{\prime} \\
\mathbf{r}_{n}^{\prime *}
\end{array}\right] s_{n}^{*}\right\}=\mathrm{E}\left\{\left[\begin{array}{c}
\mathbf{r}_{n}^{\prime} s_{n}^{*} \\
\mathbf{r}_{n}^{\prime *} s_{n}^{*}
\end{array}\right]\right\} \\
& =\left[\begin{array}{l}
\mathbf{C}_{\mathbf{r}^{\prime} s} \\
\tilde{\mathbf{C}}_{\mathbf{r}^{\prime} s}^{*}
\end{array}\right]= \begin{cases}{\left[\begin{array}{c}
\mathbf{h} \\
\mathbf{h}^{*}
\end{array}\right] ;} & \text { for BPSK } \\
{\left[\begin{array}{c}
\mathbf{h} \\
\mathbf{0}
\end{array}\right] ;} & \text { for QPSK }\end{cases}
\end{aligned}
$$

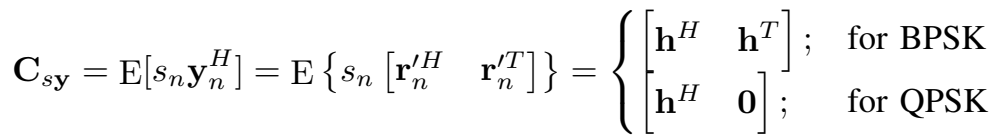

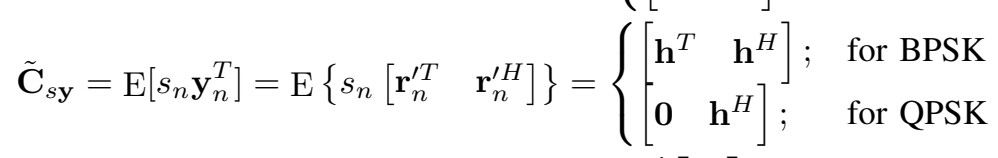

$$
\begin{aligned}
& \tilde{\mathbf{C}}_{\mathbf{y} s}=\tilde{\mathbf{C}}_{s \mathbf{y}}^{T}=\mathrm{E}\left[\mathbf{y}_{n} s_{n}\right]=\mathrm{E}\left\{\left[\begin{array}{c}
\mathbf{r}_{n}^{\prime} \\
\mathbf{r}_{n}^{\prime *}
\end{array}\right] s_{n}\right\}=\left\{\begin{array}{ll}
{\left[\begin{array}{c}
\mathbf{h} \\
\mathbf{h}^{*}
\end{array}\right] ;} & \text { for BPSK } \\
\mathbf{0} \\
\mathbf{h}^{*}
\end{array}\right] ; \quad \text { for QPSK. }
\end{aligned}
$$

For a real-valued constellation, $\tilde{\mathbf{V}}_{n}=\mathrm{E}\left\{\left[\mathbf{s}_{n}-\overline{\mathbf{s}}_{n}\right]\left[\mathbf{s}_{n}-\right.\right.$ $\left.\left.\overline{\mathbf{s}}_{n}\right]^{T}\right\}=\mathbf{V}_{n}$; for a complex-valued constellation,

$$
\begin{aligned}
& \tilde{\mathbf{V}}_{n}=\operatorname{diag}\left\{\left[\operatorname{Im}^{2}\left\{\bar{s}_{n-L+1}\right\}-\operatorname{Re}^{2}\left\{\bar{s}_{n-L+1}\right\} \quad \ldots\right.\right. \\
& \quad \operatorname{Im}^{2}\left\{\bar{s}_{n-1}\right\}-\operatorname{Re}^{2}\left\{\bar{s}_{n-1}\right\} \quad 0 \quad \operatorname{Im}^{2}\left\{\bar{s}_{n+1}\right\}-\operatorname{Re}^{2}\left\{\bar{s}_{n+1}\right\} \ldots \\
& \left.\left.\operatorname{Im}^{2}\left\{\bar{s}_{n+L-1}\right\}-\operatorname{Re}^{2}\left\{\bar{s}_{n+L-1}\right\}\right]\right\} .
\end{aligned}
$$

Each main diagonal element of $\tilde{\mathbf{V}}_{n}$ is derived as

$$
\begin{aligned}
& \mathrm{E}\left[\left(s_{n}-\bar{s}_{n}\right)^{2}\right]=\mathrm{E}\left\{\left[\left(\operatorname{Re}\left\{s_{n}\right\}+j \operatorname{Im}\left\{s_{n}\right\}\right)-\left(\operatorname{Re}\left\{\bar{s}_{n}\right\}+j \operatorname{Im}\left\{\bar{s}_{n}\right\}\right)\right]^{2}\right\} \\
& =\mathrm{E}\left[\left(\operatorname{Re}\left\{s_{n}\right\}+j \operatorname{Im}\left\{s_{n}\right\}\right)^{2}-2\left(\operatorname{Re}\left\{s_{n}\right\}+j \operatorname{Im}\left\{s_{n}\right\}\right)\right. \\
& \left.\quad \cdot\left(\operatorname{Re}\left\{\bar{s}_{n}\right\}+j \operatorname{Im}\left\{\bar{s}_{n}\right\}\right)+\left(\operatorname{Re}\left\{\bar{s}_{n}\right\}+j \operatorname{Im}\left\{\bar{s}_{n}\right\}\right)^{2}\right] \\
& =\mathrm{E}\left[\left(\operatorname{Re}\left\{s_{n}\right\}+j \operatorname{Im}\left\{s_{n}\right\}\right)^{2}\right]-2 \mathrm{E}\left[\operatorname{Re}\left\{s_{n}\right\}+j \operatorname{Im}\left\{s_{n}\right\}\right] \\
& \quad \cdot\left(\operatorname{Re}\left\{\bar{s}_{n}\right\}+j \operatorname{Im}\left\{\bar{s}_{n}\right\}\right)+\left(\operatorname{Re}\left\{\bar{s}_{n}\right\}+j \operatorname{Im}\left\{\bar{s}_{n}\right\}\right)^{2} \\
& =2 j \mathrm{E}\left[\operatorname{Re}\left\{s_{n}\right\} \operatorname{Im}\left\{s_{n}\right\}\right]-\left(\operatorname{Re}^{2}\left\{\bar{s}_{n}\right\}-\operatorname{Im}^{2}\left\{\bar{s}_{n}\right\}+2 j \operatorname{Re}\left\{\bar{s}_{n}\right\} \operatorname{Im}\left\{\bar{s}_{n}\right\}\right) \\
& =\operatorname{Im}^{2}\left\{\bar{s}_{n}\right\}-\operatorname{Re}^{2}\left\{\bar{s}_{n}\right\}+2 j\left[\mathrm{E}\left(\operatorname{Re}\left\{s_{n}\right\} \operatorname{Im}\left\{s_{n}\right\}\right)-\operatorname{Re}\left\{\bar{s}_{n}\right\} \operatorname{Im}\left\{\bar{s}_{n}\right\}\right] \\
& =\operatorname{Im}^{2}\left\{\bar{s}_{n}\right\}-\operatorname{Re}^{2}\left\{\bar{s}_{n}\right\} .
\end{aligned}
$$


The above equations are obtained based on the fact that for QPSK signals, $\operatorname{Re}^{2}\left\{s_{n}\right\}-\operatorname{Im}^{2}\left\{s_{n}\right\}=1 / 2-1 / 2=0$, and the real and imaginary parts of $\bar{s}_{n}$ correspond to two independent bits, and are thus uncorrelated ${ }^{2}$, i.e., $\operatorname{E}\left[\operatorname{Re}\left\{s_{n}\right\} \operatorname{Im}\left\{s_{n}\right\}\right]=$ $\operatorname{Re}\left\{\bar{s}_{n}\right\} \operatorname{Im}\left\{\bar{s}_{n}\right\}$.

Denoting the log-likelihood ratio (LLR) value of $s_{n}$ as $\lambda\left(s_{n}\right)=\lambda\left(x_{n}\right)+j \lambda\left(y_{n}\right)$ for QPSK signals, the soft estimate of $s_{n}$ is computed according to its LLR value $\lambda\left(s_{n}\right)$ as

$$
\bar{s}_{n}=\left\{\begin{array}{ll}
\tanh \left(\lambda\left(s_{n}\right) / 2\right) & \text { for BPSK } \\
\tanh \left[\lambda\left(x_{n}\right) / 2\right] / \sqrt{2}+j \tanh \left[\lambda\left(y_{n}\right) / 2\right] / \sqrt{2} ; & \text { for QPSK }
\end{array} .\right.
$$

At the initial stage, no priori information about $s_{n}$ is available, its LLR value is thus assumed to be zero. Therefore $\bar{s}_{n}=0$, and consequently, $\tilde{\mathbf{V}}_{n}=\mathbf{0}$ for QPSK signals, and the pseudo-autocorrelation matrix is vanishing, i.e., $\tilde{\mathbf{R}}_{n}=$ $\mathrm{E}\left[\mathbf{r}_{n}^{\prime} \mathbf{r}_{n}^{\prime T}\right]=\mathbf{H} \tilde{\mathbf{V}}_{n} \mathbf{H}^{T}=\mathbf{0}$. Note that the ISI is always improper for BPSK modulated systems since $\tilde{\mathbf{V}}_{n}=\mathbf{V}_{n}$, and $\tilde{\mathbf{R}}_{n}=\mathbf{H V}_{n} \mathbf{H}^{T} \neq \mathbf{0}$ also holds at the initial stage. At the subsequent stages, the ISI terms become improper for both real and complex-valued constellations since $\tilde{\mathbf{R}}_{n}$ is non-vanishing due to a non-vanishing matrix $\tilde{\mathbf{V}}_{n}$. In summary, we conclude that the improperness of ISI is a characteristic of the residual ISI generated at the output of the ISI cancellation process for both improper (e.g., BPSK) and proper signal constellations (e.g., QPSK), and in this case, WLP can be applied to the ISI canceled signal vector $\mathbf{r}_{n}^{\prime}$; it is also a characteristic of improper signal constellations, and WLP can be applied to the original signal vector $\mathbf{r}_{n}$ if modulation schemes such as BPSK are employed. It is worth noticing that improperness may also stem from space-time coding [14], [15]. However, this is not our concern in this work.

Some examples of improper signal constellations are 1) BPSK, which is used in the DS-UWB system [22], [23] for wireless personal area network (WPANs); 2) Gaussian minimum-shift keying (GMSK) modulation for the Global System for Mobile Communication (GSM) [24]; 3) offset quadrature amplitude modulation (OQAM) and 4) offset quadrature phase-shift keying (OQPSK), which have been adopted by the CDMA 2000 standard [25] and the mobile communication standard Universal Wireless Communications (UWC - 136) [26], respectively. In comparison, proper signal constellations (complex modulations), such as PSK/QAM, are more widely used in the current as well as in the future communication systems, e.g., in the W-CDMA [27] and broadband fixed wireless access systems [28], [29], just to name a few. In all these scenarios, utilizing the improperness of the received and/or interference canceled signals in the receiver will lead to a better performance at the expense of some increase in complexity.

Differentiating $\epsilon_{n}^{\mathrm{WL}}$ in (5) with respect to $\boldsymbol{\omega}_{n}$ results in $\frac{\partial e}{\partial \boldsymbol{\omega}_{n}}=\left(\mathbf{C}_{n} \boldsymbol{\omega}_{n}\right)^{*}-\mathbf{C}_{s \mathbf{y}}^{T}-\gamma \tilde{\mathbf{C}}_{s \mathbf{y}}^{H}$, which is set to zero to

\footnotetext{
${ }^{2}$ This is obviously true for uncoded systems. For coded systems, the interleaver breaks the bit dependency introduced by channel coding, the interleaved bits can thus be modeled as statistically independent random variables. Consequently, the real and imaginary parts of $\bar{s}_{n}$ which correspond to the consecutive coded and interleaved bits can also be regarded as uncorrelated.
}

yield the optimum vector of $\boldsymbol{\omega}_{n}$

$$
\boldsymbol{\omega}_{n}=\mathbf{C}_{n}^{-1}\left(\mathbf{C}_{s \mathbf{y}}^{H}+\gamma \tilde{\mathbf{C}}_{s \mathbf{y}}^{T}\right)=\mathbf{C}_{n}^{-1}\left(\mathbf{C}_{\mathbf{y s}}+\gamma \tilde{\mathbf{C}}_{\mathbf{y} s}\right)=\mathbf{C}_{n}^{-1}\left[\begin{array}{c}
\mathbf{h} \\
\gamma \mathbf{h}^{*}
\end{array}\right] .
$$

For the proposed WL equalizer, the augmented autocorrelation matrix $\mathbf{C}_{n}$ expressed in (6) which gives a complete second order description of $\mathbf{r}_{n}^{\prime}$ is used for deriving the equalizer filter coefficient vector $\boldsymbol{\omega}_{n}$; whereas for the conventional linear MMSE algorithm, the filter coefficient vector $\mathbf{w}_{n}$ is calculated using only the autocorrelation of the observation $\mathbf{R}_{n}=$ $\mathrm{E}\left[\mathbf{r}_{n}^{\prime} \mathbf{r}_{n}^{\prime H}\right]$ and the cross-correlation between the observation and desired signal $\mathbf{C}_{\mathbf{r}^{\prime} s}=\mathrm{E}\left[\mathbf{r}_{n}^{\prime} s_{n}^{*}\right]$, i.e., $\mathbf{w}_{n}=\mathbf{R}_{n}^{-1} \mathbf{C}_{\mathbf{r}^{\prime} s}$. The pseudo-autocorrelation matrix $\tilde{\mathbf{R}}_{n}$ is implicitly assumed to be zero. However, as shown previously, $\tilde{\mathbf{R}}_{n}$ is non-vanishing except at the initial stage for QPSK signals, hence omitting $\tilde{\mathbf{R}}_{n}$ would lead to sub-optimum solutions.

The WL MMSE filter output can be expressed as $z_{n}=$ $\mu_{n} s_{n}+\mu_{n}^{\prime} s_{n}^{*}+\eta_{n}$, where the combined noise and residual interference $\eta_{n}$ can be approximated as a Gaussian random variable [20], [30]. In [16], $\eta_{n}$ is regarded as a proper random process since $\mathrm{E}\left[\eta_{n}^{2}\right]=0$ for a sufficiently large spreading factor. However, as will become evident later on, this treatment is sub-optimum for the equalization algorithm under study. Next, we present two schemes to derive the LLR values for $x_{n}$ and $y_{n}$ for QPSK systems based on the assumption that the interference-plus-noise term $\eta_{n}$ at the output of the WL filter output is either a proper or an improper random process.

\section{A. Scheme 1}

The first scheme is based on the common assumption that $\eta_{n}$ is proper. In this case, the second order statistic of the zeromean Gaussian random variable $\eta_{n}$ is completely characterized by its variance $N_{\eta}=\mathrm{E}\left[\left|\eta_{n}\right|^{2}\right]$. The parameters $\mu_{n}, \mu_{n}^{\prime}, N_{\eta}$ can be determined by taking expectation with respect to the interfering symbols and the channel noise

$$
\begin{aligned}
\mu_{n} & =\mathrm{E}\left\{z_{n} s_{n}^{*}\right\}=\boldsymbol{\omega}_{n}^{H} \mathrm{E}\left[\mathbf{y}_{n} s_{n}^{*}\right]=\boldsymbol{\omega}_{n}^{H} \mathbf{C}_{\mathbf{y} s} \\
\mu_{n}^{\prime} & =\mathrm{E}\left\{z_{n} s_{n}\right\}=\boldsymbol{\omega}_{n}^{H} \mathrm{E}\left[\mathbf{y}_{n} s_{n}\right]=\boldsymbol{\omega}_{n}^{H} \tilde{\mathbf{C}}_{\mathbf{y} s} \\
N_{\eta} & =\mathrm{E}\left[\left|\eta_{n}\right|^{2}\right]=\mathrm{E}\left[\left|z_{n}-\mu_{n} s_{n}-\mu_{n}^{\prime} s_{n}^{*}\right|^{2}\right] \\
& =\mathrm{E}\left[\left(z_{n}-\mu_{n} s_{n}-\mu_{n}^{\prime} s_{n}^{*}\right)\left(z_{n}^{*}-\mu_{n}^{*} s_{n}^{*}-\mu_{n}^{\prime *} s_{n}\right)\right] \\
& =\mathrm{E}\left\{\left|z_{n}\right|^{2}\right\}-\left|\mu_{n}\right|^{2}-\left|\mu_{n}^{\prime}\right|^{2}=\mu_{n}^{*}+\gamma \mu_{n}^{\prime *}-\left|\mu_{n}\right|^{2}-\left|\mu_{n}^{\prime}\right|^{2}
\end{aligned}
$$

The above equation holds since $z_{n}=\boldsymbol{\omega}_{n}^{H} \mathbf{y}_{n}$ and $\boldsymbol{\omega}_{n}=$ $\mathbf{C}_{n}^{-1}\left(\mathbf{C}_{\mathbf{y} s}+\gamma \tilde{\mathbf{C}}_{\mathbf{y} s}\right)$, therefore,

$$
\begin{aligned}
\mathrm{E}\left\{\left|z_{n}\right|^{2}\right\} & =\mathrm{E}\left\{\boldsymbol{\omega}_{n}^{H} \mathbf{y}_{n} \mathbf{y}_{n}^{H} \boldsymbol{\omega}_{n}\right\}=\boldsymbol{\omega}_{n}^{H} \mathbf{C}_{n} \boldsymbol{\omega}_{n} \\
& =\left(\mathbf{C}_{\mathbf{y} s}^{H}+\gamma \tilde{\mathbf{C}}_{\mathbf{y} s}^{H}\right) \mathbf{C}_{n}^{-1} \mathbf{C}_{n} \boldsymbol{\omega}_{n} \\
& =\left(\mathbf{C}_{\mathbf{y} s}^{H}+\gamma \tilde{\mathbf{C}}_{\mathbf{y} s}^{H}\right) \boldsymbol{\omega}_{n}=\mu_{n}^{*}+\gamma \mu_{n}^{\prime *}
\end{aligned}
$$

After computing the values of $\mu_{n}, \mu_{n}^{\prime}$ and $N_{\eta}$, the conditional PDF of the equalizer output can be obtained as

$$
f\left(z_{n} \mid s_{n}=s_{M}\right)=\frac{1}{\pi N_{\eta}} \exp \left(-\frac{\left|z_{n}-\mu_{n} s_{M}-\mu_{n}^{\prime} s_{M}^{*}\right|^{2}}{N_{\eta}}\right)
$$




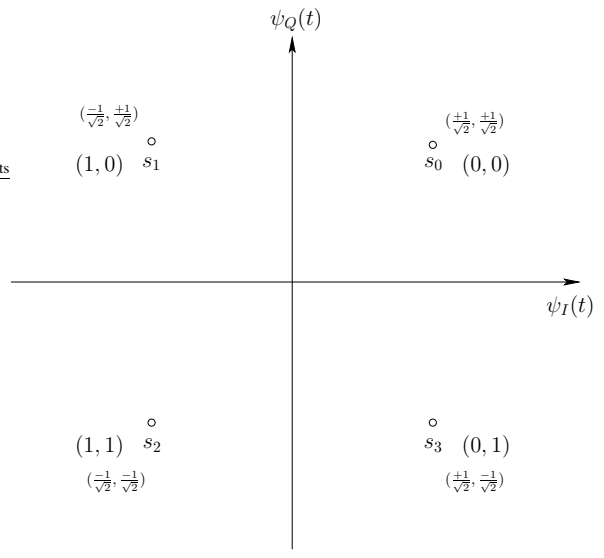

Fig. 1. QPSK constellation and bit-to-symbol mapping.

and the LLR value of $x_{n}$ can thus be computed as

$$
\begin{aligned}
\lambda\left(x_{n}\right) & =\ln \frac{f\left(z_{n} \mid x_{n}=0\right)}{f\left(z_{n} \mid x_{n}=1\right)}=\ln \frac{f\left(z_{n} \mid s_{n}=s_{0}\right)+f\left(z_{n} \mid s_{n}=s_{3}\right)}{f\left(z_{n} \mid s_{n}=s_{1}\right)+f\left(z_{n} \mid s_{n}=s_{2}\right)} \\
& \approx \ln \frac{\exp \left(-\left|z_{n}-\mu_{n} s_{n}^{+}-\mu_{n}^{\prime} s_{n}^{+*}\right|^{2} / N_{\eta}\right)}{\exp \left(-\left|z_{n}-\mu_{n} s_{n}^{-}-\mu_{n}^{\prime} s_{n}^{-*}\right|^{2} / N_{\eta}\right)} \\
& =\frac{2}{N_{\eta}} \operatorname{Re}\left\{\left(\mu_{n} s_{n}^{+*} z_{n}+\mu_{n}^{\prime} s_{n}^{+} z_{n}\right)-\left(\mu_{n} s_{n}^{-*} z_{n}+\mu_{n}^{\prime} s_{n}^{-} z_{n}\right)\right\}
\end{aligned}
$$

where $s_{n}^{+}$denotes the QPSK symbol corresponding to $\max \left\{f\left(z_{n} \mid s_{n}=s_{0}\right), f\left(z_{n} \mid s_{n}=s_{3}\right)\right\}$, and $s_{n}^{-}$denotes the QPSK symbol corresponding to $\max \left\{f\left(z_{n} \mid s_{n}=\right.\right.$ $\left.\left.s_{1}\right), f\left(z_{n} \mid s_{n}=s_{2}\right)\right\}$ since the real part of the symbols $s_{0}, s_{3}$ corresponds to 0 , and the real part of the symbols $s_{1}, s_{2}$ corresponds to 1 as shown in Fig. 1. The dual maxima rule [31] is used in (10) utilizing the fact that one term usually dominates each sum.

Similarly,

$$
\begin{aligned}
& \lambda\left(y_{n}\right)=\ln \frac{f\left(z_{n} \mid s_{n}=s_{0}\right)+f\left(z_{n} \mid s_{n}=s_{1}\right)}{f\left(z_{n} \mid s_{n}=s_{2}\right)+f\left(z_{n} \mid s_{n}=s_{3}\right)} \\
& \approx \frac{2}{N_{\eta}} \operatorname{Re}\left\{\left(\mu_{n} s_{n}^{+*} z_{n}+\mu_{n}^{\prime} s_{n}^{+} z_{n}\right)-\left(\mu_{n} s_{n}^{-*} z_{n}+\mu_{n}^{\prime} s_{n}^{-} z_{n}\right)\right\},
\end{aligned}
$$

where $s_{n}^{+}$denotes the QPSK symbol corresponding to $\max \left\{f\left(z_{n} \mid s_{n}=s_{0}\right), f\left(z_{n} \mid s_{n}=s_{1}\right)\right\}$, and $s_{n}^{-}$denotes the QPSK symbol corresponding to $\max \left\{f\left(z_{n} \mid s_{n}=\right.\right.$ $\left.\left.s_{2}\right), f\left(z_{n} \mid s_{n}=s_{3}\right)\right\}$ since the imaginary part of the symbols $s_{0}, s_{1}$ corresponds to 0 , and the imaginary part of the symbols $s_{2}, s_{3}$ corresponds to 1 as shown in Fig. 1 .

Then we use Equ. (7) to convert LLRs to soft symbol estimate $\bar{s}_{n}$, which is needed by the equalizer for the interference cancellation at the next iteration.

\section{B. Scheme 2}

The second scheme takes into account the improperness of $\eta_{n}$, and utilizes the fact that $\tilde{N}_{\eta}=\mathrm{E}\left[\eta_{n}^{2}\right] \neq 0$. According to the definition,

$$
\begin{aligned}
\tilde{N}_{\eta} & =\mathrm{E}\left[\eta_{n}^{2}\right]=\mathrm{E}\left[\left(z_{n}-\mu_{n} s_{n}-\mu_{n}^{\prime} s_{n}^{*}\right)^{2}\right] \\
& =\mathrm{E}\left[\left(z_{n}-\mu_{n} s_{n}-\mu_{n}^{\prime} s_{n}^{*}\right)\left(z_{n}-\mu_{n} s_{n}-\mu_{n}^{\prime} s_{n}^{*}\right)\right] \\
& =\mathrm{E}\left\{z_{n}^{2}\right\}-2 \mu_{n} \mu_{n}^{\prime}=\mathrm{E}\left\{\boldsymbol{\omega}_{n}^{H} \mathbf{y}_{n} \mathbf{y}_{n}^{T} \boldsymbol{\omega}_{n}^{*}\right\}-2 \mu_{n} \mu_{n}^{\prime} \\
& =\boldsymbol{\omega}_{n}^{H} \tilde{\mathbf{C}}_{n} \boldsymbol{\omega}_{n}^{*}-2 \mu_{n} \mu_{n}^{\prime} .
\end{aligned}
$$

Equ. (11) holds since $\boldsymbol{\omega}_{n}^{H} \mathbf{y}=\mathbf{y}^{T} \boldsymbol{\omega}_{n}^{*}$. The matrix $\tilde{\mathbf{C}}_{n}$ is computed as

$$
\begin{aligned}
\tilde{\mathbf{C}}_{n} & =\mathrm{E}\left\{\mathbf{y}_{n} \mathbf{y}_{n}^{T}\right\}=\mathrm{E}\left\{\left[\begin{array}{c}
\mathbf{r}_{n}^{\prime} \\
\mathbf{r}_{n}^{\prime *}
\end{array}\right]\left[\begin{array}{cc}
\mathbf{r}_{n}^{\prime T} & \mathbf{r}_{n}^{\prime H}
\end{array}\right]\right\}=\left[\begin{array}{cc}
\tilde{\mathbf{R}}_{n} & \mathbf{R}_{n} \\
\mathbf{R}_{n}^{*} & \tilde{\mathbf{R}}_{n}^{*}
\end{array}\right] \\
& =\left[\begin{array}{cc}
\mathbf{H} \tilde{\mathbf{V}}_{n} \mathbf{H}^{T} & \mathbf{H V}_{n} \mathbf{H}^{H}+N_{0} \mathbf{I} \\
\mathbf{H}^{*} \mathbf{V}_{n} \mathbf{H}^{T}+N_{0} \mathbf{I} & \mathbf{H}^{*} \tilde{\mathbf{V}}_{n}^{*} \mathbf{H}^{H}
\end{array}\right] .
\end{aligned}
$$

Let us denote $z_{n}=z_{n}^{r}+j z_{n}^{i}, s_{n}=s_{n}^{r}+j s_{n}^{i}$, and $\eta_{n}=$ $\eta_{n}^{r}+j \eta_{n}^{i}$. The filter output $z_{n}=\mu_{n} s_{n}+\mu_{n}^{\prime} s_{n}^{*}+\eta_{n}$ can be re-written as

$$
\underbrace{\left[\begin{array}{c}
z_{n}^{r} \\
z_{n}^{i}
\end{array}\right]}_{\mathbf{t}_{n}}=\underbrace{\left[\begin{array}{c}
\left(\mu_{n}+\mu_{n}^{\prime}\right) s_{n}^{r} \\
\left(\mu_{n}-\mu_{n}^{\prime}\right) s_{n}^{i}
\end{array}\right]}_{\mathbf{d}_{n}}+\underbrace{\left[\begin{array}{c}
\eta_{n}^{r} \\
\eta_{n}^{i}
\end{array}\right]}_{\mathbf{i}_{n}} .
$$

Since the probability distribution of a complex random variable or vector is a joint distribution of its real and imaginary part, we have

$$
\begin{aligned}
& f\left(z_{n} \mid s_{n}\right)=f\left(\mathbf{t}_{n} \mid \mathbf{d}_{n}\right) \\
& =\frac{1}{2 \pi \sqrt{\operatorname{det} \mathbf{W}_{n}}} \exp \left(-\frac{1}{2}\left(\mathbf{t}_{n}-\mathbf{d}_{n}\right)^{H} \mathbf{W}_{n}^{-1}\left(\mathbf{t}_{n}-\mathbf{d}_{n}\right)\right)
\end{aligned}
$$

where the covariance matrix of the Gaussian noise is $\mathbf{W}_{n}=$ $\mathrm{E}\left[\mathbf{i}_{n} \mathbf{i}_{n}^{H}\right]$. Let us define

$$
\begin{aligned}
\tilde{\mathbf{t}}_{n}=\left[\begin{array}{c}
z_{n} \\
z_{n}^{*}
\end{array}\right] ; & \tilde{\mathbf{d}}_{n}=\left[\begin{array}{c}
\mu_{n} s_{n}+\mu_{n}^{\prime} s_{n}^{*} \\
\left(\mu_{n} s_{n}+\mu_{n}^{\prime} s_{n}^{*}\right)^{*}
\end{array}\right] ; \\
\tilde{\mathbf{i}}_{n}=\left[\begin{array}{c}
\eta_{n} \\
\eta_{n}^{*}
\end{array}\right] ; & \widetilde{\mathbf{W}}_{n}=\mathrm{E}\left[\tilde{\mathbf{i}}_{n} \tilde{\mathbf{i}}_{n}^{H}\right],
\end{aligned}
$$

and a mapping matrix [4], [32]

$$
\mathbf{J}=\frac{1}{\sqrt{2}}\left[\begin{array}{cc}
1 & j \\
1 & -j
\end{array}\right],
$$

which is an unitary matrix since $\mathbf{J} \mathbf{J}^{H}=\mathbf{J}^{H} \mathbf{J}=\mathbf{I}$. It can be easily shown that $\mathbf{t}_{n}-\mathbf{d}_{n}=\frac{1}{\sqrt{2}} \mathbf{J}^{H}\left(\tilde{\mathbf{t}}_{n}-\tilde{\mathbf{d}}_{n}\right)$, and $\tilde{\mathbf{i}}_{n}=\sqrt{2} \mathbf{J} \mathbf{i}_{n}$. The PDF expressed by (12) can thus be reformed as

$$
\begin{aligned}
& f\left(z_{n} \mid s_{n}\right)=\frac{1}{2 \pi \sqrt{\operatorname{det} \mathbf{W}_{n}}} \exp \left(-\frac{1}{4}\left(\tilde{\mathbf{t}}_{n}-\tilde{\mathbf{d}}_{n}\right)^{H} \mathbf{J} \mathbf{W}_{n}^{-1} \mathbf{J}^{H}\left(\tilde{\mathbf{t}}_{n}-\tilde{\mathbf{d}}_{n}\right)\right) \\
& =\frac{1}{2 \pi \sqrt{\operatorname{det} \mathbf{W}_{n}}} \exp \left(-\frac{1}{4}\left(\tilde{\mathbf{t}}_{n}-\tilde{\mathbf{d}}_{n}\right)^{H}\left(\mathbf{J} \mathbf{W}_{n} \mathbf{J}^{H}\right)^{-1}\left(\tilde{\mathbf{t}}_{n}-\tilde{\mathbf{d}}_{n}\right)\right) \\
& =\frac{1}{2 \pi \sqrt{\operatorname{det} \mathbf{W}_{n}}} \exp \left(-\frac{1}{2}\left(\tilde{\mathbf{t}}_{n}-\tilde{\mathbf{d}}_{n}\right)^{H} \widetilde{\mathbf{W}}_{n}^{-1}\left(\tilde{\mathbf{t}}_{n}-\tilde{\mathbf{d}}_{n}\right)\right),
\end{aligned}
$$

where

$$
\begin{aligned}
\widetilde{\mathbf{W}}_{n} & =\mathrm{E}\left[\tilde{\mathbf{i}}_{n} \tilde{\mathbf{i}}_{n}^{H}\right]=\mathrm{E}\left\{\left[\begin{array}{l}
\eta_{n} \\
\eta_{n}^{*}
\end{array}\right]\left[\begin{array}{ll}
\eta_{n}^{*} & \eta_{n}
\end{array}\right]\right\} \\
& =\mathrm{E}\left\{\left[\begin{array}{ll}
\eta_{n} \eta_{n}^{*} & \eta_{n} \eta_{n} \\
\eta_{n}^{*} \eta_{n}^{*} & \eta_{n}^{*} \eta_{n}
\end{array}\right]\right\}=\left[\begin{array}{cc}
N_{\eta} & \tilde{N}_{\eta} \\
\tilde{N}_{\eta}^{*} & N_{\eta}
\end{array}\right] .
\end{aligned}
$$

The third equality in (13) follows from the fact that

$$
\begin{aligned}
\mathbf{J W}_{n} \mathbf{J}^{H} & =\mathbf{J} \mathrm{E}\left[\mathbf{i}_{n} \mathbf{i}_{n}^{H}\right] \mathbf{J}^{H}=\mathrm{E}\left[\left(\mathbf{J i}_{n}\right)\left(\mathbf{J i}_{n}\right)^{H}\right] \\
& =\frac{1}{2} \mathrm{E}\left[\tilde{\mathbf{i}}_{n} \tilde{\mathbf{i}}_{n}^{H}\right]=\frac{1}{2} \widetilde{\mathbf{W}}_{n} .
\end{aligned}
$$


The LLR value of $x_{n}$ can thus be computed as

$$
\begin{aligned}
& \lambda\left(x_{n}\right)=\ln \frac{f\left(z_{n} \mid x_{n}=0\right)}{f\left(z_{n} \mid x_{n}=1\right)}=\ln \frac{f\left(z_{n} \mid s_{n}^{r}=+1 / \sqrt{2}\right)}{f\left(z_{n} \mid s_{n}^{r}=-1 / \sqrt{2}\right)} \\
& \approx \ln \frac{\exp \left(-\frac{1}{2}\left(\tilde{\mathbf{t}}_{n}-\tilde{\mathbf{d}}_{n}^{+}\right)^{H} \widetilde{\mathbf{W}}_{n}^{-1}\left(\tilde{\mathbf{t}}_{n}-\tilde{\mathbf{d}}_{n}^{+}\right)\right)}{\exp \left(-\frac{1}{2}\left(\tilde{\mathbf{t}}_{n}-\tilde{\mathbf{d}}_{n}^{-}\right)^{H} \widetilde{\mathbf{W}}_{n}^{-1}\left(\tilde{\mathbf{t}}_{n}-\tilde{\mathbf{d}}_{n}^{-}\right)\right)} \\
& =-\frac{1}{2}\left[\left(\tilde{\mathbf{t}}_{n}-\tilde{\mathbf{d}}_{n}^{+}\right)^{H} \widetilde{\mathbf{W}}_{n}^{-1}\left(\tilde{\mathbf{t}}_{n}-\tilde{\mathbf{d}}_{n}^{+}\right)\right. \\
& \left.\quad-\left(\tilde{\mathbf{t}}_{n}-\tilde{\mathbf{d}}_{n}^{-}\right)^{H} \widetilde{\mathbf{W}}_{n}^{-1}\left(\tilde{\mathbf{t}}_{n}-\tilde{\mathbf{d}}_{n}^{-}\right)\right],
\end{aligned}
$$

where $\tilde{\mathbf{d}}_{n}^{+}$denotes the vector $\tilde{\mathbf{d}}_{n}$ corresponding to

$\max \left\{f\left(\tilde{\mathbf{t}}_{n} \mid s_{n}^{r}=\frac{+1}{\sqrt{2}}, s_{n}^{i}=\frac{+1}{\sqrt{2}}\right), f\left(\tilde{\mathbf{t}}_{n} \mid s_{n}^{r}=\frac{+1}{\sqrt{2}}, s_{n}^{i}=\frac{-1}{\sqrt{2}}\right)\right\} ;$

which is equivalent to $\max \left\{f\left(\tilde{\mathbf{t}}_{n} \mid s_{n}=s_{0}\right), f\left(\tilde{\mathbf{t}}_{n} \mid s_{n}=s_{3}\right)\right\}$, and $\tilde{\mathbf{d}}_{n}^{-}$denotes the vector $\tilde{\mathbf{d}}_{n}$ corresponding to

$\max \left\{f\left(\tilde{\mathbf{t}}_{n} \mid s_{n}^{r}=\frac{-1}{\sqrt{2}}, s_{n}^{i}=\frac{+1}{\sqrt{2}}\right), f\left(\tilde{\mathbf{t}}_{n} \mid s_{n}^{r}=\frac{-1}{\sqrt{2}}, s_{n}^{i}=\frac{-1}{\sqrt{2}}\right)\right\}$

which is equivalent to $\max \left\{f\left(\tilde{\mathbf{t}}_{n} \mid s_{n}=s_{1}\right), f\left(\tilde{\mathbf{t}}_{n} \mid s_{n}=s_{2}\right)\right\}$.

Similarly,

$$
\begin{aligned}
\lambda\left(y_{n}\right)= & \ln \frac{f\left(z_{n} \mid y_{n}=0\right)}{f\left(z_{n} \mid y_{n}=1\right)}=\ln \frac{f\left(z_{n} \mid s_{n}^{i}=+1 / \sqrt{2}\right)}{f\left(z_{n} \mid s_{n}^{i}=-1 / \sqrt{2}\right)} \\
\approx- & \frac{1}{2}\left[\left(\tilde{\mathbf{t}}_{n}-\tilde{\mathbf{d}}_{n}^{+}\right)^{H} \widetilde{\mathbf{W}}_{n}^{-1}\left(\tilde{\mathbf{t}}_{n}-\tilde{\mathbf{d}}_{n}^{+}\right)\right. \\
& \left.\quad-\left(\tilde{\mathbf{t}}_{n}-\tilde{\mathbf{d}}_{n}^{-}\right)^{H} \widetilde{\mathbf{W}}_{n}^{-1}\left(\tilde{\mathbf{t}}_{n}-\tilde{\mathbf{d}}_{n}^{-}\right)\right]
\end{aligned}
$$

where $\tilde{\mathbf{d}}_{n}^{+}$denotes the vector $\tilde{\mathbf{d}}_{n}$ corresponding to $\max \left\{f\left(\tilde{\mathbf{t}}_{n} \mid s_{n}=s_{0}\right), f\left(\tilde{\mathbf{t}}_{n} \mid s_{n}=s_{1}\right)\right\}$, and $\tilde{\mathbf{d}}_{n}^{-}$denotes the vector $\tilde{\mathbf{d}}_{n}$ corresponding to $\max \left\{f\left(\tilde{\mathbf{t}}_{n} \mid s_{n}=s_{3}\right), f\left(\tilde{\mathbf{t}}_{n} \mid s_{n}=\right.\right.$ $\left.\left.s_{2}\right)\right\}$.

\section{NumERICAL RESUlTS}

In this section, we compare the performance of the proposed schemes with that of the WL MMSE and DFE equalizers introduced in [5] and the iterative linear MMSE equalizer introduced in [19]-[21]. During each Monte-Carlo run, the block size is set to 10000 information bits, which corresponds to 5000 QPSK or 10000 BPSK symbols. The noise variance $N_{0}$ and the complex channel coefficients are assumed to be known to the receiver. Both time-varying and static channels are tested. For the time-varying channel, we choose the SUI-3 fixed wireless access channel introduced in [29], [33]. The channel coefficients vary from one data block to another, however, they are assumed to remain constant during the transmission of one block of data, due to the slowly time varying nature of the SUI-3 channel ${ }^{3}$. For the static channel, we use a 5-tap channel with impulse response $h[n]=(2-$ $0.4 j) \delta[n]+(1.5+1.8 j) \delta[n-1]+\delta[n-2]+(1.2-1.3 j) \delta[n-$ $3]+(0.8+1.6 j) \delta[n-4]$. The total channel gain is normalized so that $P=\sum_{n=0}^{4}|h[n]|^{2}=1$.

\footnotetext{
${ }^{3}$ In fact, the proposed algorithm only requires the channel to be constant during the transmission of $2 L-1$ symbols (where $L$ is the number of channel taps), i.e., during the transmission of symbols $s_{n-L+1}, \ldots, s_{n+L-1}$ in the vector $\mathbf{s}_{n}$ expressed by (2).
}

Fig. 2 shows the performance of different equalization schemes for BPSK signals transmitted over the SUI-3 channel. The results are averaged over at least 500 channel realizations. The 10-tap WL MMSE equalizer proposed in [5] outperforms its linear counterparts by over $1 \mathrm{~dB}$ at BERs between $10^{-2}$ and $10^{-3}$. The use of decision feedback can further improve the performance since it is observed that the 10-tap WL DFE (6 feedforward taps and 4 feedback taps) yields better results than the 10-tap WL MMSE. The performance of the conventional DFE lies between the WL MMSE and the WL DFE. More gain is obtained by applying WLP to linear MMSE filtering than to DFE. Equalizers with length greater than 10 taps are also tested and are shown to have a similar performance to the 10-tap equalizers. The figure also shows that the proposed WL equalizer at the 3rd stage outperforms the WL DFE by $0.8 \mathrm{~dB}$ at $\mathrm{BER}=10^{-3}$. It is observed that most of the gains are obtained at the 2 nd and the 3rd stages with the proposed iterative equalization scheme, for which the parameter $\gamma$ in (8) is set to be 1. As discussed in Section II, the ISI is improper for BPSK systems with and without interference cancellation. This is verified by Fig. 2.b), which compares the performance between the proposed scheme and the iterative linear MMSE scheme introduced in [19]-[21]. The topmost curve represents the initial stage equalization and the bottommost curve represents the 4th stage equalization. We noticed significant performance improvement by applying WLP at the first iteration (no interference cancellation has taken place yet), as well as at the second iteration (interference cancellation has been performed). However, the performance gap becomes much smaller when the algorithms reach convergence at the 4th iteration.

Fig. 3 shows the impact of the parameter $\gamma$ on the performance of the proposed equalization schemes for QPSK signals transmitted over the SUI-3 channel. The curve is plotted for different values of $\gamma$ at the 5th equalization stage, and $E_{b} / N_{0}$ is set to $20 \mathrm{~dB}$. The results are averaged over at least 500 channel realizations so that the proposed procedure would experience a variety of different channel conditions. Both Figs. 3.a) and 3.b) indicate that the performance of the WL scheme 2 is independent of $\gamma$. For the WL scheme 1, as illustrated by Fig. 3.a), when $\gamma<1$, the optimum value is $\gamma=0$, which is the solution proposed in [16]; when choosing in the region $\gamma>1$, Fig. 3.b) indicates that there seem to be infinite number of choices because the performance of the scheme 1 becomes insensitive to the choice of $\gamma$ when it goes beyond a certain value (e.g., $\gamma=15$ ). The second scheme outperforms the first one at all values of $\gamma$, indicating that ignoring the improperness of the residual interference-plusnoise at the filter output renders performance loss, and the scheme accounting for its improperness is always optimized regardless of the value of $\gamma$.

One can see that the choice of $\gamma=1$ leads to the worst performance for first scheme (it also does not work for the second scheme, which is not shown in the figure). This is in contrast to systems with real-valued constellations, for which $\gamma=1$ is appropriate. The reason is that $\boldsymbol{\omega}_{n}[1]=$ $\boldsymbol{\omega}_{n}^{*}[0]$ when choosing $\gamma=1$, and it can be shown that $\mu_{n}=\mu_{n}^{\prime}$ in such a case. Therefore, the equalizer output 


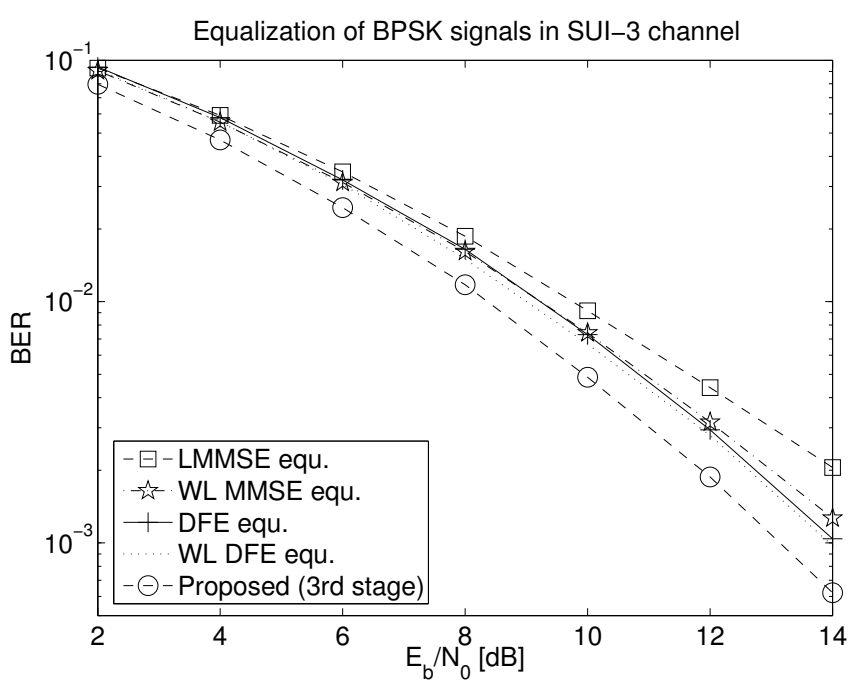

(a)

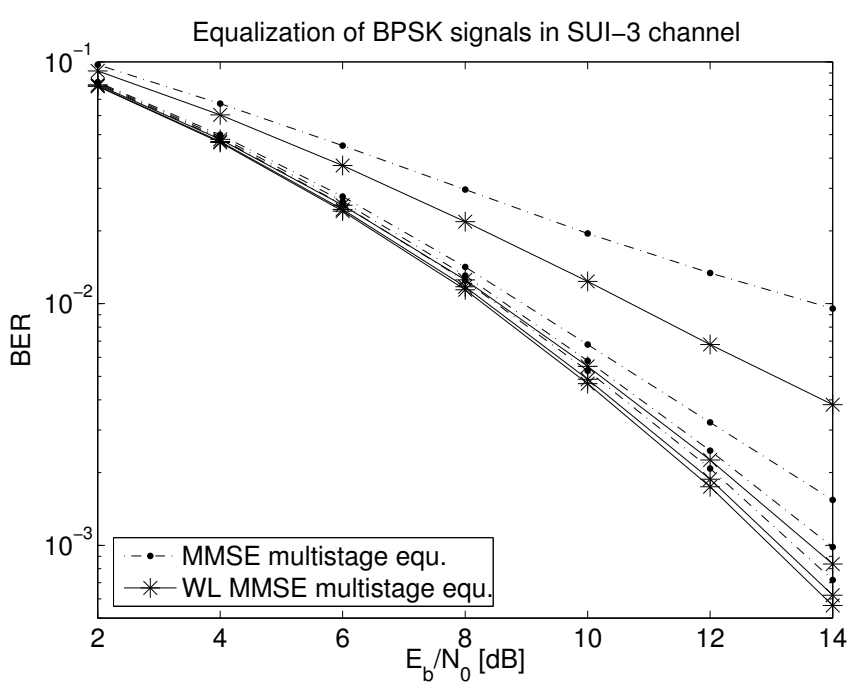

(b)

Fig. 2. Comparison of different equalization schemes for BPSK signals in SUI-3 channel.

becomes $z_{n}=\mu_{n} s_{n}+\mu_{n}^{\prime} s_{n}^{*}+\eta_{n}=2 \mu_{n} \operatorname{Re}\left\{s_{n}\right\}+\eta_{n}$, which means the decision statistic does not contain any information about the imaginary part of the symbol $s_{n}$. Consequently, the second bit corresponding to the imaginary part of the QPSK symbol cannot be correctly detected. In order to tackle this problem, we can adopt the method suggested by the MaxSINR approach derived in the appendix, i.e., using $\gamma=1$ and $\gamma=-1$ for making the decision for the first bit and second bit corresponding to the real and imaginary parts of the QPSK symbol, respectively. The points marked '*' in plot a) show the performance for the Max-SINR approach. Since it decodes the two bits of QPSK symbols independently without utilizing the correlation between the real and imaginary parts of the filter output (failing to exploit the improperness), it is therefore a suboptimum solution compared to the scheme exploiting improperness as shown by the result in the figure.

Fig. 4 shows the performance comparison between the proposed schemes and the iterative linear MMSE scheme introduced in [19]-[21] for QPSK signals transmitted over the SUI-3 channel. It takes 4-5 stages for all the algorithms to converge. Upon convergence, the proposed schemes outperform the linear MMSE scheme by $2-3 \mathrm{~dB}$ at BER around $10^{-3}$. Compared to the initial equalization stage, the subsequent equalization stages achieve much better performance, and most gains are obtained at the 2 nd and 3 rd stages. The WL scheme 2 outperforms the WL scheme 1 by $1 \mathrm{~dB}$ at $\mathrm{BER}=10^{-3}$. In contrast to the systems with BPSK systems, all the algorithms have identical performance at the initial stage due to the fact that the improperness of ISI for QPSK signals is only a characteristic of interference cancellation as shown in Section II $\left(\tilde{\mathbf{R}}_{n}=\mathbf{0}\right.$ for QPSK signalling at the initial stage), thus no improvement can be achieved by WLP. At the following cancellation stages, the ISI canceled signal becomes improper, and exploitation of the improperness of the
ISI results in better performance.

The three schemes are compared for the 5-tap static channel in Fig. 5. After the system reaches convergence at the 6th stage, performance gains of $1.1 \mathrm{~dB}$ and $1.8 \mathrm{~dB}$ compared to that of the linear MMSE scheme are observed by applying the WL scheme 1 and scheme 2 , respectively, at BER $=10^{-4}$. As indicated by Fig. 4 and Fig. 5, the proposed equalizers do not achieve much performance gain at low SNR. This is due to the fact that the proper noise is dominant at low SNR; whereas the advantage of exploiting the improper ISI becomes evident when the SNR increases. Fig. 5.b) also shows clear superiority of the WL scheme 2 over the conventional MMSE (10 taps) and DFE (6 feedforward taps and 4 feedback taps) equalizers. Note that no gain can be obtained by the WL MMSE or the WL DFE since the improperness of ISI only comes from interference cancellation for QPSK signals.

\section{CONCLUSIONS}

In this paper, we introduced an iterative widely linear approach to equalization of signals transmitted over frequency selective channels. The proposed algorithm is generic in the sense that it is applicable for both real and complex signalling formats. The improper nature of the ISI is analyzed and different behavior evident for real and complex signals has been revealed by our simulations using BPSK and QPSK modulation. In the latter case, we proposed two variants of the widely linear equalization algorithm, both of which utilize the improperness of the ISI canceled signal at the input of the WL MMSE filter. However, the first scheme is based on the common assumption that the residual interference-plusnoise at the filter output is proper, whereas the second scheme takes into consideration the improperness of the filter output. The results indicate that the algorithm exploiting improperness at both input and output of the WL filter leads to the best 


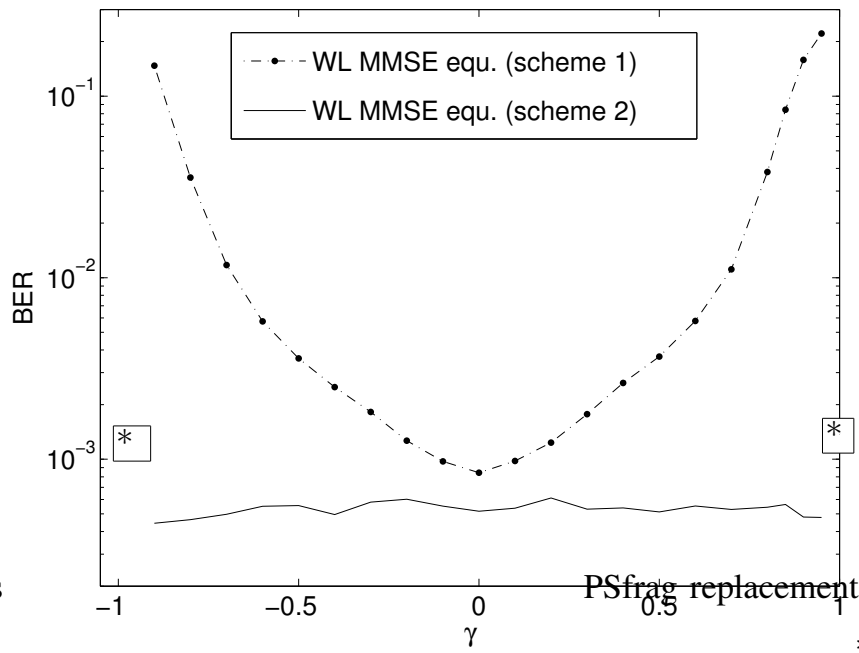

(a) BER as a function of $\gamma(\gamma \leq 1)$.

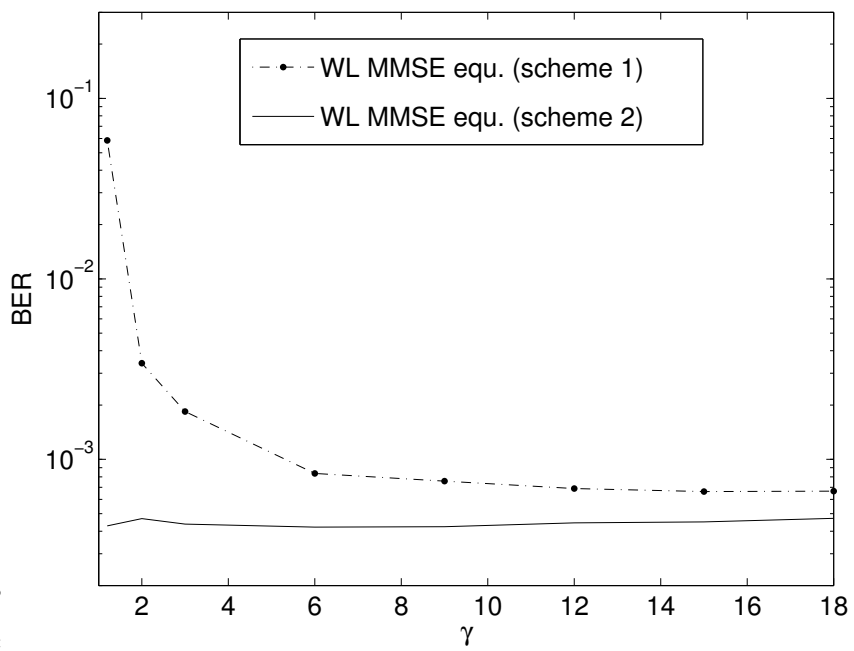

(b) BER as a function of $\gamma(\gamma \geq 1)$.

Fig. 3. Impact of $\gamma$ on the performance of the proposed equalization schemes for QPSK signals. In plot (a), the markers with ' $*$ ' indicate the performance of the Max-SINR approach at $\gamma=1$ and $\gamma=-1$.

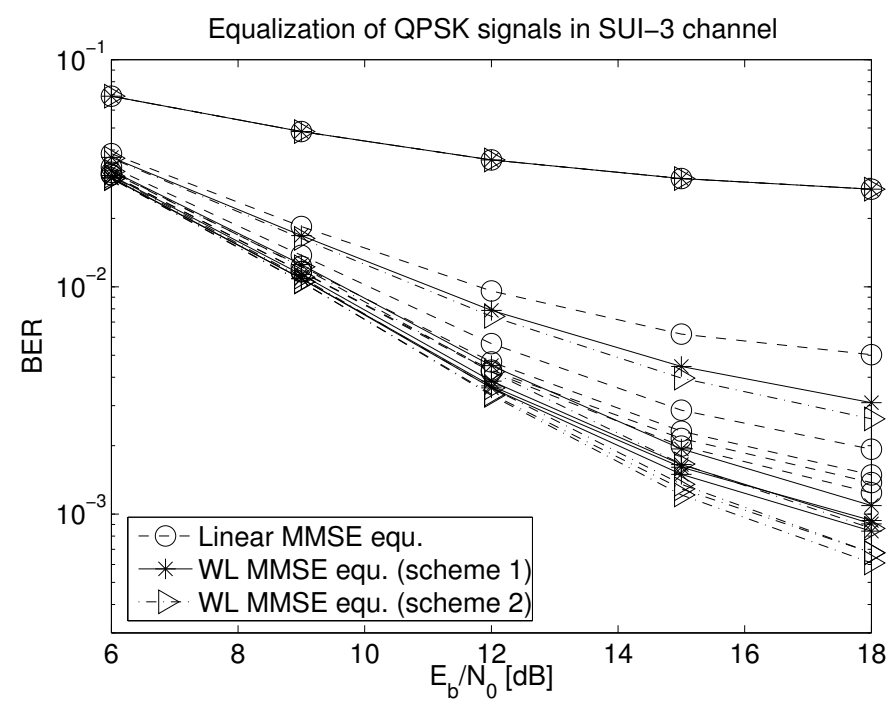

Fig. 4. Performance of the proposed equalization for QPSK signals in SUI-3 channel $(\gamma=15)$. BER for different stages of the iterative process is shown.

performance. This is in contrast to the previously proposed widely multiuser detector in CDMA systems, for which the improperness of the filter output can be neglected.

\section{ACKNOWLEDGMENT}

The authors would like to thank the anonymous reviewers whose insightful comments have significantly improved the quality of this work.

\section{REFERENCES}

[1] P. Schreier, L. Scharf, C. Mullis. "Detection and estimation of improper complex random signals", IEEE Transactions on Information Theory, vol. 51, no. 1, pp. 306-312, January 2005.
[2] F. Neeser, J. Massey. "Proper complex random processes with applications to information theory", IEEE Transactions on Information Theory, vol. 39, no. 4, pp. 1293-1302, July 1993.

[3] B. Picinbono, P. Chevalier. "Widely linear estimation with complex data", IEEE Transactions on Signal Processing, vol. 43, no. 8, pp. 2030-2033, August 1995.

[4] P. Schreier, L. Scharf. "Second-order analysis of improper complex random vectors and processes", IEEE Transactions on Signal Processing, vol. 51, no. 3, pp. 714-725, March 2003.

[5] W. Gerstacker, R. Schober, A. Lampe. "Receivers with widely linear processing for frequency-selective channels". IEEE Transactions on Communications, vol. 51, no. 9, pp. 1512-1523, Sept. 2003.

[6] P. Schreier, L. Scharf, C. Mullis. "A unified approach to performance comparisons between linear and widely linear processing". Proc. IEEE Workshop on Statistical Signal Processing, pp. 114-117, Sept. 2003.

[7] S. Buzzi, M. Lops, A. Tulino. "A new family of MMSE multiuser receivers for interference suppression in DS/CDMA systems employing BPSK modulation". IEEE Transactions on Communications, vol. 49, no. 1, pp. 154-167, Jan. 2001.

[8] A. Mirbagheri, K. Plataniotis, S. Pasupathy. "An enhanced widely linear CDMA receiver with OQPSK modulation", IEEE Transactions on Communications, vol. 54, no. 2, pp. 261-272, Feb. 2006.

[9] Y. Yoon, H. Kim. "An efficient blind multiuser detection for improper DS/CDMA signals". IEEE Transactions on Vehicular Technology, vol. 55, no. 2, pp. 572-582, March 2006.

[10] D. Darsena, G. Geli, L. Paura, F. Verde. "Widely linear equalization and blind channel identification for interference-contaminated multicarrier systems". IEEE Transactions on Signal Processing, vol. 53, no. 3, pp. 1163-1177, March 2005.

[11] A. Parihar, L. Lampe, R. Schober, C. Leung. "Equalization for DSUWB systems - Part I: BPSK modulation". Proc. IEEE Transactions on Communications, vol. 55, no. 6, pp. 1164-1173, June 2007.

[12] A. Parihar, L. Lampe, R. Schober, C. Leung. "Equalization for 4BOK DS-UWB systems". Proc. IEEE International Conference on Communications, pp. 1482-1487, June 2006.

[13] S. Buzzi, M. Lops, S. Sardellitti. "Widely linear reception strategies for layered space-time wireless communications". IEEE Transactions on Signal Processing, vol. 54, no. 6, pp. 2252-2262, June 2006.

[14] W. Gerstacker, F. Obernosterer, R. Schober, A. Lehmann, A. Lampe, P. Gunreben. "Equalization concepts for Alamouti's space-time block code", IEEE Transactions on Communications, vol. 52, no. 7, pp. 11781190, July 2004.

[15] M. Witzke, S. Baro, J. Hagenauer. "Iterative detection of generalized coded MIMO signals using a widely linear detector", IEEE Global Telecommunications Conference, vol. 4, pp. 1821-1825, Dec. 2003. 


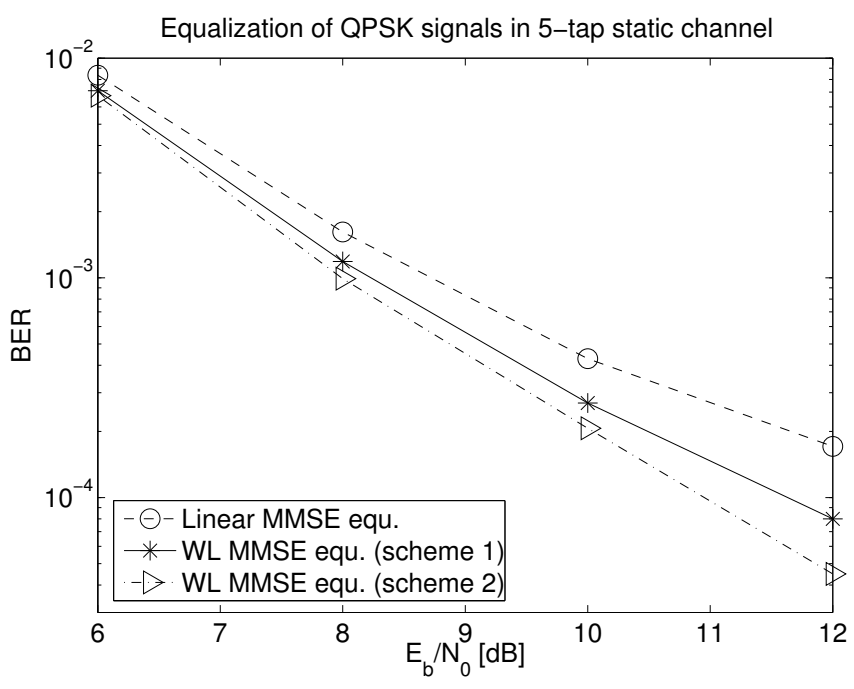

(a)

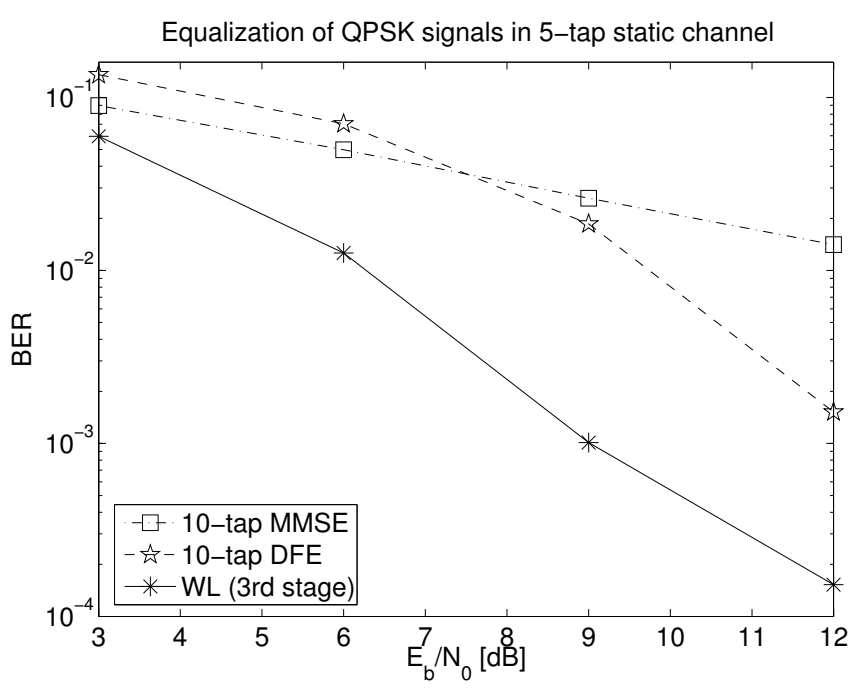

(b)

Fig. 5. Comparison of different equalization schemes for QPSK signals in 5-tap static channel $(\gamma=0)$. In plot (a), the curves represent the performance at the 6th stage for all the schemes. In plot (b), BER of the WL scheme 2 at the 3rd stage is shown.

[16] A. Lampe, R. Scholber, W. Gerstacker, J. Huber. "A novel iterative multiuser detector for complex modulation schemes", IEEE Journal on Selected Areas in Communications, vol. 20, no. 2, pp. 339-350, Feb. 2002.

[17] D. Mattera, L. Paura, F. Sterle. "Widely linear MMSE equaliser for MIMO linear time-dispersive channel", Electronic Letters, vol. 39, no. 20, pp. 1481-1482, Oct. 2003.

[18] D. Mattera, L. Paura, F. Sterle. "Widely linear decision-feedback equalizer for time-dispersive linear MIMO channel", IEEE Transactions on Signal Processing, vol. 53, no. 7, pp. 2525-2536, July 2005.

[19] K. Narayanan. "Turbo equalization". In Wiley Encyclopedia of Telecommunications, vol. 5, pp. 2716-2727, 2002.

[20] X. Wang, H. Poor. "Iterative (turbo) soft interference cancellation and decoding for coded CDMA", IEEE Transactions on Communications, vol. 47, pp. 1046-1061, July 1999.

[21] M. Tuchler, R. Koetter, A. Singer. "Turbo equalization: principles and new results", IEEE Transactions on Communications, vol. 50, pp. 754767, May 2002.

[22] "IEEE 802.15 WPAN High Rate Alternative PHY Task Group 3a (TG3a)". Available at http://www.ieee802.org/15/pub/TG3a.html.

[23] R. Fisher, R. Kohno, M. McLaughlin, M. Welbourn. "IEEE P802.15/04/0137r4", Jan. 2005.

[24] "GSM Recommendation 05.04, Modulation", Feb. 1992.

[25] "Physical layer standard for cdma 2000 spread spectrum systems", May 2002. Available at $h t t p: / / w w w .3 g p p 2 . o r g$

[26] "Study of the required geographic coordination distance between a HAPS TDMA system and adjacent system IMT-2000 terrestrial mobile stations operating in the same frequency band". ITU Radiocommunications Study Groups, Doc. 8-1/368-E, 1999.

[27] 3G TS 25.213. 3rd generation partnership project: technical specification group radio access networks; Spreading and modulation (FDD), V4.2.0, Dec. 2001.

[28] H. Bolcskei. "Fixed broadband wireless access: state of the art, challenges, and future directions". IEEE Communication Magazine, pp. 100-108, Jan. 2001

[29] IEEE 802.16 working group on broadband wireless access standards. available at http://grouperieee.org/groups/802/16/. 2002.

[30] V. Poor, S. Verdu. "Probability of error in MMSE multiuser detection", IEEE Transactions on Communications, vol. 43, pp. 858-971, May 1997.

[31] A. Viterbi. "An intuitive justification and a simplified implementation of the MAP decoder for convolutional codes". IEEE Journals on Selected Areas in Communications, vol. 16, no. 2, pp. 260-264, Feb. 1998

[32] A. Bos. "The multivariate complex normal distribution - a generaliza- tion". IEEE Transactions on Information Theory, vol. 41, no. 2, pp. 537-539, March 1995.

[33] Channel models for fixed wireless applications. available at http://www.esqube.com/ieee802.16channelModels.pdf

\section{APPENDIX}

\section{Maximum SINR solution}

One way of choosing an appropriate value of $\gamma$ is to maximize the signal-to-interference-plus-noise ratio (SINR) at the filter output $z_{n}=\mu_{n} s_{n}+\mu_{n}^{\prime} s_{n}^{*}+\eta_{n}$, which can be expressed as

$$
\operatorname{SINR}=\frac{\left|\mu_{n}(\gamma)\right|^{2}+\left|\mu_{n}^{\prime}(\gamma)\right|^{2}}{N_{\eta}(\gamma)}=\frac{\left|\mu_{n}(\gamma)\right|^{2}+\left|\mu_{n}^{\prime}(\gamma)\right|^{2}}{\mathrm{E}\left\{\left|z_{n}\right|^{2}\right\}-\left|\mu_{n}(\gamma)\right|^{2}-\left|\mu_{n}^{\prime}(\gamma)\right|^{2}},
$$

where

$$
\begin{aligned}
\mu_{n} & =\mathrm{E}\left\{z_{n} s_{n}^{*}\right\}=\boldsymbol{\omega}_{n}^{H} \mathrm{E}\left[\mathbf{y} s_{n}^{*}\right]=\boldsymbol{\omega}_{n}^{H} \mathbf{C}_{\mathbf{y} s} \\
\mu_{n}^{\prime} & =\mathrm{E}\left\{z_{n} s_{n}\right\}=\boldsymbol{\omega}_{n}^{H} \mathrm{E}\left[\mathbf{y} s_{n}\right]=\boldsymbol{\omega}_{n}^{H} \tilde{\mathbf{C}}_{\mathbf{y} s} ; \\
\boldsymbol{\omega}_{n} & =\mathbf{C}_{n}^{-1}\left[\begin{array}{c}
\mathbf{h} \\
\gamma \mathbf{h}^{*}
\end{array}\right] ; \quad \mathbf{C}_{\mathbf{y} s}=\left[\begin{array}{l}
\mathbf{h} \\
\mathbf{0}
\end{array}\right] ; \quad \tilde{\mathbf{C}}_{\mathbf{y} s}=\left[\begin{array}{c}
\mathbf{0} \\
\mathbf{h}^{*}
\end{array}\right] .
\end{aligned}
$$

The solutions of the this approach can be obtained by differentiating SINR with respect to $\gamma$ and setting it to zero. The matrix $\mathbf{C}_{n}$ is a hermitian matrix, i.e., $\mathbf{C}_{n}^{H}=\mathbf{C}_{n}$. Therefore, $\left(\mathbf{C}_{n}^{-1}\right)^{H}=\left(\mathbf{C}_{n}^{H}\right)^{-1}=$ $\mathbf{C}_{n}^{-1}$. Let us denote $\mathbf{C}_{n}^{-1}=\left[\begin{array}{ll}\mathbf{C}_{00} & \mathbf{C}_{01} \\ \mathbf{C}_{10} & \mathbf{C}_{11}\end{array}\right]$. Using the block matrix inverse formula

$$
\left[\begin{array}{ll}
\mathbf{A} & \mathbf{B} \\
\mathbf{C} & \mathbf{D}
\end{array}\right]^{-1}=\left[\begin{array}{cc}
\left(\mathbf{A}-\mathbf{B D}^{-1} \mathbf{C}\right)^{-1} & -\left(\mathbf{A}-\mathbf{B D}^{-1} \mathbf{C}\right)^{-1} \mathbf{B D}^{-1} \\
-\left(\mathbf{D}-\mathbf{C A}^{-1} \mathbf{B}\right)^{-1} \mathbf{C A}^{-1} & \left(\mathbf{D}-\mathbf{C A}^{-1} \mathbf{B}\right)^{-1}
\end{array}\right] .
$$

For the matrix $\mathbf{C}_{n}=\left[\begin{array}{cc}\mathbf{R} & \tilde{\mathbf{R}} \\ \tilde{\mathbf{R}}^{*} & \mathbf{R}^{*}\end{array}\right]$ defined in (6), we have

$$
\mathbf{C}_{n}^{-1}=\left[\begin{array}{cc}
\mathbf{R} & \tilde{\mathbf{R}} \\
\tilde{\mathbf{R}}^{*} & \mathbf{R}^{*}
\end{array}\right]^{-1}
$$

$$
=\left[\begin{array}{cc}
\left(\mathbf{R}-\tilde{\mathbf{R}} \mathbf{R}^{*-1} \tilde{\mathbf{R}}^{*}\right)^{-1} & -\left(\mathbf{R}-\tilde{\mathbf{R}} \mathbf{R}^{*-1} \tilde{\mathbf{R}}^{*}\right)^{-1} \tilde{\mathbf{R}} \mathbf{R}^{*-1} \\
-\left(\mathbf{R}^{*}-\tilde{\mathbf{R}}{ }^{*} \mathbf{R}^{-1} \tilde{\mathbf{R}}\right)^{-1} \tilde{\mathbf{R}}^{*} \mathbf{R}^{-1} & \left(\mathbf{R}^{*}-\tilde{\mathbf{R}}^{*} \mathbf{R}^{-1} \tilde{\mathbf{R}}\right)^{-1}
\end{array}\right]
$$

$\mathbf{C}_{00}=\left(\mathbf{R}-\tilde{\mathbf{R}} \mathbf{R}^{*-1} \tilde{\mathbf{R}}^{*}\right)^{-1} ; \quad \mathbf{C}_{01}=-\left(\mathbf{R}-\tilde{\mathbf{R}} \mathbf{R}^{*-1} \tilde{\mathbf{R}}^{*}\right)^{-1} \tilde{\mathbf{R}} \mathbf{R}^{*-1} ;$

$\mathbf{C}_{10}=-\left(\mathbf{R}^{*}-\tilde{\mathbf{R}}^{*} \mathbf{R}^{-1} \tilde{\mathbf{R}}\right)^{-1} \tilde{\mathbf{R}}^{*} \mathbf{R}^{-1} ; \quad \mathbf{C}_{11}=\left(\mathbf{R}^{*}-\tilde{\mathbf{R}}^{*} \mathbf{R}^{-1} \tilde{\mathbf{R}}\right)^{-1}$ 
It is obvious to see from the above equations that $\mathbf{C}_{11}=\mathbf{C}_{00}^{*}$ and $\mathbf{C}_{10}=\mathbf{C}_{01}^{*}$. The exact expressions of $\left|\mu_{n}(\gamma)\right|^{2},\left|\mu_{n}^{\prime}(\gamma)\right|^{2}$ and $\mathrm{E}\left\{\left|z_{n}\right|^{2}\right\}$ can be obtained as

$$
\begin{aligned}
&\left|\mu_{n}(\gamma)\right|^{2}=\boldsymbol{\omega}_{n}^{H} \mathbf{C}_{\mathbf{y} s} \mathbf{C}_{\mathbf{y} s}^{H} \boldsymbol{\omega}_{n} \\
&= {\left[\begin{array}{ll}
\mathbf{h}^{H} & \gamma \mathbf{h}^{T}
\end{array}\right]\left[\begin{array}{ll}
\mathbf{C}_{00} & \mathbf{C}_{01} \\
\mathbf{C}_{10} & \mathbf{C}_{11}
\end{array}\right]\left[\begin{array}{l}
\mathbf{h} \\
\mathbf{0}
\end{array}\right]\left[\begin{array}{ll}
\mathbf{h}^{H} & \mathbf{0}
\end{array}\right]\left[\begin{array}{ll}
\mathbf{C}_{00} & \mathbf{C}_{01} \\
\mathbf{C}_{10} & \mathbf{C}_{11}
\end{array}\right]\left[\begin{array}{c}
\mathbf{h} \\
\gamma \mathbf{h}^{*}
\end{array}\right] } \\
&= \mathbf{h}^{H} \mathbf{C}_{00} \mathbf{h h}^{H} \mathbf{C}_{00} \mathbf{h}+\gamma\left(\mathbf{h}^{T} \mathbf{C}_{10} \mathbf{h h}^{H} \mathbf{C}_{00} \mathbf{h}+\mathbf{h}^{H} \mathbf{C}_{00} \mathbf{h} \mathbf{h}^{H} \mathbf{C}_{01} \mathbf{h}^{*}\right) \\
&+\gamma^{2} \mathbf{h}^{T} \mathbf{C}_{10} \mathbf{h h}^{H} \mathbf{C}_{01} \mathbf{h}^{*} ; \\
&\left|\mu_{n}^{\prime}(\gamma)\right|^{2}=\boldsymbol{\omega}_{n}^{H} \tilde{\mathbf{C}}_{\mathbf{y} s} \tilde{\mathbf{C}}_{\mathbf{y} s}^{H} \boldsymbol{\omega}_{n} \\
&= {\left[\begin{array}{ll}
\mathbf{h}^{H} & \gamma \mathbf{h}^{T}
\end{array}\right]\left[\begin{array}{ll}
\mathbf{C}_{00} & \mathbf{C}_{01} \\
\mathbf{C}_{10} & \mathbf{C}_{11}
\end{array}\right]\left[\begin{array}{c}
\mathbf{0} \\
\mathbf{h}^{*}
\end{array}\right]\left[\begin{array}{ll}
\mathbf{0} & \mathbf{h}^{T}
\end{array}\right]\left[\begin{array}{ll}
\mathbf{C}_{00} & \mathbf{C}_{01} \\
\mathbf{C}_{10} & \mathbf{C}_{11}
\end{array}\right]\left[\begin{array}{c}
\mathbf{h} \\
\gamma \mathbf{h}^{*}
\end{array}\right] } \\
&= \mathbf{h}^{H} \mathbf{C}_{01} \mathbf{h}^{*} \mathbf{h}^{T} \mathbf{C}_{10} \mathbf{h} \\
&+\gamma\left(\mathbf{h}^{H} \mathbf{C}_{01} \mathbf{h}^{*} \mathbf{h}^{T} \mathbf{C}_{11} \mathbf{h}^{*}+\mathbf{h}^{T} \mathbf{C}_{11} \mathbf{h}^{*} \mathbf{h}^{T} \mathbf{C}_{10} \mathbf{h}\right) \\
&+\gamma^{2} \mathbf{h}^{T} \mathbf{C}_{11} \mathbf{h}^{*} \mathbf{h}^{T} \mathbf{C}_{11} \mathbf{h}^{*} ; \\
& \mathrm{E}\left\{\left|z_{n}\right|^{2}\right\}=\mathrm{E}\left\{\boldsymbol{\omega}_{n}^{H} \mathbf{y}_{n} \mathbf{y}_{n}^{H} \boldsymbol{\omega}_{n}\right\}=\boldsymbol{\omega}_{n}^{H} \mathbf{C}_{n} \boldsymbol{\omega}_{n} \\
&=\left(\mathbf{C}_{\mathbf{y} s}^{H}+\gamma \tilde{\mathbf{C}}_{\mathbf{y} s}^{H}\right) \mathbf{C}_{n}^{-1} \mathbf{C}_{n} \boldsymbol{\omega}_{n}=\left(\mathbf{C}_{\mathbf{y} s}^{H}+\gamma \tilde{\mathbf{C}}_{\mathbf{y} s}^{H}\right) \boldsymbol{\omega}_{n} \\
&= {\left[\mathbf{h}^{H} \quad \gamma \mathbf{h}^{T}\right]\left[\begin{array}{ll}
\mathbf{C}_{00} & \mathbf{C}_{01} \\
\mathbf{C}_{10} & \mathbf{C}_{11}
\end{array}\right]\left[\begin{array}{c}
\mathbf{h} \\
\gamma \mathbf{h}^{*}
\end{array}\right] } \\
&= \mathbf{h}^{H} \mathbf{C}_{00} \mathbf{h}+\gamma\left(\mathbf{h}^{T} \mathbf{C}_{10} \mathbf{h}+\mathbf{h}^{H} \mathbf{C}_{01} \mathbf{h}^{*}\right)+\gamma^{2} \mathbf{h}^{T} \mathbf{C}_{11} \mathbf{h}^{*},
\end{aligned}
$$

where $\mathrm{E}\left\{\left|z_{n}\right|^{2}\right\}$ is derived using the fact that $z_{n}=\boldsymbol{\omega}_{n}^{H} \mathbf{y}$ and $\boldsymbol{\omega}_{n}=$ $\mathbf{C}_{n}^{-1}\left(\mathbf{C}_{\mathbf{y} s}+\gamma \tilde{\mathbf{C}}_{\mathbf{y} s}\right)$. Denote

$$
\begin{aligned}
\operatorname{SINR} & =\frac{\left|\mu_{n}(\gamma)\right|^{2}+\left|\mu_{n}^{\prime}(\gamma)\right|^{2}}{N_{\eta}(\gamma)}=\frac{a(\gamma)}{b(\gamma)} \\
a(\gamma) & =\left|\mu_{n}(\gamma)\right|^{2}+\left|\mu_{n}^{\prime}(\gamma)\right|^{2}=a_{0}+a_{1} \gamma+a_{2} \gamma^{2} \\
b(\gamma) & =N_{\eta}(\gamma)=\mathrm{E}\left\{\left|z_{n}\right|^{2}\right\}-\left|\mu_{n}(\gamma)\right|^{2}-\left|\mu_{n}^{\prime}(\gamma)\right|^{2} \\
& =\mathrm{E}\left\{\left|z_{n}\right|^{2}\right\}-a(\gamma)=b_{0}+b_{1} \gamma+b_{2} \gamma^{2} .
\end{aligned}
$$

From (18), we know that

$$
\begin{aligned}
a_{0}= & \mathbf{h}^{H} \mathbf{C}_{01} \mathbf{h}^{*} \mathbf{h}^{T} \mathbf{C}_{10} \mathbf{h}+\mathbf{h}^{H} \mathbf{C}_{00} \mathbf{h} \mathbf{h}^{H} \mathbf{C}_{00} \mathbf{h} \\
a_{1}= & \mathbf{h}^{T} \mathbf{C}_{10} \mathbf{h h}^{H} \mathbf{C}_{00} \mathbf{h}+\mathbf{h}^{H} \mathbf{C}_{00} \mathbf{h h}^{H} \mathbf{C}_{01} \mathbf{h}^{*} \\
& +\mathbf{h}^{H} \mathbf{C}_{01} \mathbf{h}^{*} \mathbf{h}^{T} \mathbf{C}_{11} \mathbf{h}^{*}+\mathbf{h}^{T} \mathbf{C}_{11} \mathbf{h}^{*} \mathbf{h}^{T} \mathbf{C}_{10} \mathbf{h} \\
a_{2}= & \mathbf{h}^{T} \mathbf{C}_{10} \mathbf{h} \mathbf{h}^{H} \mathbf{C}_{01} \mathbf{h}^{*}+\mathbf{h}^{T} \mathbf{C}_{11} \mathbf{h}^{*} \mathbf{h}^{T} \mathbf{C}_{11} \mathbf{h}^{*} \\
b_{0}= & \mathbf{h}^{H} \mathbf{C}_{00} \mathbf{h}-a_{0} \\
b_{1}= & \mathbf{h}^{T} \mathbf{C}_{10} \mathbf{h}+\mathbf{h}^{H} \mathbf{C}_{01} \mathbf{h}^{*}-a_{1} \\
b_{2}= & \mathbf{h}^{T} \mathbf{C}_{11} \mathbf{h}^{*}-a_{2} .
\end{aligned}
$$

Since $\mathbf{C}_{11}=\mathbf{C}_{00}^{*}$ and $\mathbf{C}_{10}=\mathbf{C}_{01}^{*}$ as shown in (17), consequently, $a_{2}=a_{0}^{*}$ and $b_{2}=b_{0}^{*}$. It is observed that they are real-valued parameters, and the imaginary part of these variables is negligible. Therefore, $a_{2}=a_{0}$ and $b_{2}=b_{0}$. Differentiating SINR $=\frac{a(\gamma)}{b(\gamma)}$ with respect to $\gamma$ yields

$$
\frac{\partial}{\partial \gamma}\left(\frac{a(\gamma)}{b(\gamma)}\right)=\left[b(\gamma) \frac{\partial a(\gamma)}{\partial \gamma}-a(\gamma) \frac{\partial b(\gamma)}{\partial \gamma}\right] / b(\gamma)^{2}
$$

The optimum values of $\gamma$ is obtained by setting (20) to zero, leading to the solution

$$
b(\gamma) \frac{\partial a(\gamma)}{\partial \gamma}=a(\gamma) \frac{\partial b(\gamma)}{\partial \gamma}
$$

By (19), we have

$$
\begin{aligned}
& b(\gamma) \frac{\partial a(\gamma)}{\partial \gamma}=\left(b_{0}+\gamma b_{1}+\gamma^{2} b_{2}\right)\left(a_{1}+2 \gamma a_{2}\right) \\
& a(\gamma) \frac{\partial b(\gamma)}{\partial \gamma}=\left(a_{0}+\gamma a_{1}+\gamma^{2} a_{2}\right)\left(b_{1}+2 \gamma b_{2}\right) .
\end{aligned}
$$

The equation $b(\gamma) \frac{\partial a(\gamma)}{\partial \gamma}=a(\gamma) \frac{\partial b(\gamma)}{\partial \gamma}$ is then equivalent to $\left(a_{1} b_{2}-\right.$ $\left.a_{2} b_{1}\right) \gamma^{2}+2\left(a_{0} b_{2}-a_{2} b_{0}\right) \gamma+a_{0} b_{1}-a_{1} b_{0}=0$. With $a_{2}=a_{0}, b_{2}=$ $b_{0}$, it reduces to $\left(a_{1} b_{2}-a_{2} b_{1}\right) \gamma^{2}-\left(a_{1} b_{2}-a_{2} b_{1}\right)=0$, leading to the final solution $\gamma_{\mathrm{opt}}= \pm 1$.

In what follows, we explain how the solution can be used for deriving LLR values. It can be easily shown that $\mu_{n}^{\prime}=$ $\left\{\begin{array}{ll}\mu_{n} & \text { if } \gamma=1, \\ -\mu_{n} & \text { if } \gamma=-1\end{array}\right.$. Therefore, $N_{\eta}=\mu_{n}^{*}+\gamma \mu_{n}^{\prime *}-\left|\mu_{n}\right|^{2}-\left|\mu_{n}^{\prime}\right|^{2}=$ $2 \mu_{n}^{*}-2\left|\mu_{n}\right|^{2}$. Let us denote the LLR value of $s_{n}$ as $\lambda\left(s_{n}\right)=$ $\lambda\left(x_{n}\right)+j \lambda\left(y_{n}\right)$ for QPSK signals, the solution $\gamma=1$ is used for deriving the LLR value of $x_{n}$, i.e.,

$$
\begin{aligned}
\boldsymbol{\omega}_{0} & =\mathbf{C}_{n}^{-1}\left[\begin{array}{c}
\mathbf{h} \\
\gamma \mathbf{h}^{*}
\end{array}\right]=\mathbf{C}_{n}^{-1}\left[\begin{array}{c}
\mathbf{h} \\
\mathbf{h}^{*}
\end{array}\right] ; \\
z_{n}[0] & =\boldsymbol{\omega}_{0}^{H} \mathbf{y} ; \quad \mu_{n}[0]=\boldsymbol{\omega}_{0}^{H} \mathbf{C}_{\mathbf{y} s} .
\end{aligned}
$$

Denoting $z_{n}[0]=\mu_{n}[0] s_{n}+\mu_{n}[0] s_{n}^{*}+\eta_{n}=2 \mu_{n}[0] \operatorname{Re}\left\{s_{n}\right\}+\eta_{n}$, and approximating $\eta_{n}$ as Gaussian random variable [20], [30], yield the conditional PDF

$$
f\left(z_{n}[0] \mid s_{n}=s_{M}\right)=\frac{1}{\pi N_{\eta}} \exp \left(-\frac{\left|z_{n}[0]-2 \mu_{n}[0] \operatorname{Re}\left\{s_{M}\right\}\right|^{2}}{N_{\eta}}\right),
$$

and the LLR value of $x_{n}$ can be computed as

$$
\begin{aligned}
\lambda\left(x_{n}\right) & =\ln \frac{f\left(z_{n}[0] \mid x_{n}=0\right)}{f\left(z_{n}[0] \mid x_{n}=1\right)}=\ln \frac{f\left(z_{n}[0] \mid s_{n}=s_{0}\right)+f\left(z_{n}[0] \mid s_{n}=s_{3}\right)}{f\left(z_{n}[0] \mid s_{n}=s_{1}\right)+f\left(z_{n}[0] \mid s_{n}=s_{2}\right)} \\
& =\ln \frac{2 \exp \left(-\left|z_{n}[0]-2 \mu_{n}[0] / \sqrt{2}\right|^{2} / N_{\eta}\right)}{2 \exp \left(-\left|z_{n}[0]+2 \mu_{n}[0] / \sqrt{2}\right|^{2} / N_{\eta}\right)}=\frac{8 \mu_{n}[0] \operatorname{Re}\left\{z_{n}[0]\right\}}{\sqrt{2} N_{\eta}} .
\end{aligned}
$$
i.e.,

The solution $\gamma=-1$ is used for deriving the LLR value of $y_{n}$,

$$
\begin{aligned}
\boldsymbol{\omega}_{1} & =\mathbf{C}_{n}^{-1}\left[\begin{array}{c}
\mathbf{h} \\
\gamma \mathbf{h}^{*}
\end{array}\right]=\mathbf{C}_{n}^{-1}\left[\begin{array}{c}
\mathbf{h} \\
-\mathbf{h}^{*}
\end{array}\right] ; \\
z_{n}[1] & =\boldsymbol{\omega}_{1}^{H} \mathbf{y} ; \quad \mu_{n}[1]=\boldsymbol{\omega}_{1}^{H} \mathbf{C}_{\mathbf{y} s} .
\end{aligned}
$$

Since $z_{n}[1]=\mu_{n}[1] s_{n}-\mu_{n}[1] s_{n}^{*}+\eta_{n}=2 \mu_{n}[1] \operatorname{Im}\left\{s_{n}\right\} j+\eta_{n}$, the conditional PDF and LLR value of $y_{n}$ can be derived similarly as

$$
\begin{aligned}
f\left(z_{n}[1] \mid s_{n}=s_{M}\right) & =\frac{1}{\pi N_{\eta}} \exp \left(-\frac{\left|z_{n}[1]-2 \mu_{n}[1] \operatorname{Im}\left\{s_{M}\right\} j\right|^{2}}{N_{\eta}}\right) ; \\
\lambda\left(y_{n}\right) & =\ln \frac{f\left(z_{n}[1] \mid y_{n}=0\right)}{f\left(z_{n}[1] \mid y_{n}=1\right)} \\
& =\ln \frac{2 \exp \left(-\left|z_{n}[1]-2 \mu_{n}[1] j / \sqrt{2}\right|^{2} / N_{\eta}\right)}{2 \exp \left(-\left|z_{n}[1]+2 \mu_{n}[1] j / \sqrt{2}\right|^{2} / N_{\eta}\right)} \\
& =\frac{8 \mu_{n}[1] \operatorname{Im}\left\{z_{n}[1]\right\}}{\sqrt{2} N_{\eta}} .
\end{aligned}
$$

Note that this Max-SINR approach suggests independent processing of the real and the imaginary parts of the filtered signal, the correlation between $\operatorname{Re}\left\{\eta_{n}\right\}$ and $\operatorname{Im}\left\{\eta_{n}\right\}$ is not exploited in any way. It is therefore suboptimum, and the scheme described in Section II-B is not applicable in this case. 